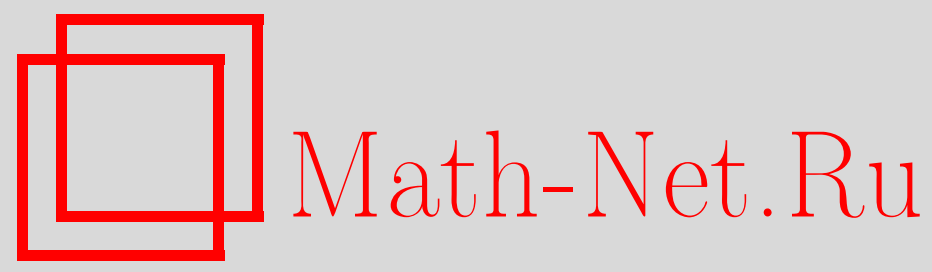

В. В. Чистяков, О многозначных отображениях конечной обобщенной вариации, Матем. заметки, 2002, том 71, выпуск 4, 611-632

DOI: https://doi.org/10.4213/mzm372

Использование Общероссийского математического портала Math-Net.Ru подразумевает, что вы прочитали и согласны с пользовательским соглашением http://www.mathnet.ru/rus/agreement

Параметры загрузки:

IP : 54.157 .27 .8

26 апреля 2023 г., 06:35:51






\section{О МНОГОЗНАЧНЫХ ОТОБРАЖЕНИЯХ КОНЕЧНОЙ ОБОБЩЕННОЙ ВАРИАЦИИ}

\section{В.В. Чистяков}

Изучаются отображения, действующие из вещественного промежутка в метрические пространства и имеющие конечную обобщенную вариацию в смысле ЖорданаРисса-Орлича. Устанавливаются вложения функциональных пространств, структура отображений, скачки вариации, принцип выбора Хелли. Показано, что компактнозначное многозначное отображение конечной обобщенной вариации относительно хаусдорфовой метрики обладает регулярной селекцией конечной обобщенной вариации. Представлен результат о существовании селекций, сохраняющих свойства многозначного отображения, которое определено на прямом произведении промежутка и топологического пространства и имеет конечную обобщенную вариацию по первой переменной и полунепрерывно сверху по второй.

Библиограффия: 35 названий.

1. Введение. Многозначное отображсение из множества $E$ в множество $X$ - это отображение $F$, ставящее в соответствие каждому элементу $t \in E$ некоторое (непустое) множество $F(t) \subset X$, называемое образом $t$. График $F$ определяется как множество $\operatorname{Gr}(F)=\{(t, x) \in E \times X \mid x \in F(t)\}$. Селекиией многозначного отображения $F$ назьвается любое однозначное отображение $f: E \rightarrow X$, для которого $f(t) \in F(t)$ для всех $t \in E$.

Множество работ (например, [1]-[5]) посвящено непрерывньп и измеримым селекциям при весьма общих предположениях относительно $E$ и $X$. Непрерьвные селекции существуют для многозначных отображений, как правило, имеющих выпуклые образы. Примеры [1], [6] показьвают, что без этого условия выпуклости непрерьвные селекции могут не существовать даже для непрерьвных отображений из отрезка прямой в компактные подмножества $\mathbb{R}^{2}$ или для липшицевых отображений из $\mathbb{R}^{3}$ в компактные подмножества пространства $\mathbb{R}^{3}$. Интерес представляют селекции, наследующие глобальные (зависящие от всей области определения) характеристические свойства исходного многозначного отображения. Их мы будем называть регулярными.

Пусть $\mathrm{c}(X)$ обозначает семейство всех непустых компактньх подмножеств метрического пространства $(X, d)$. Хаусдорфова метрика на $\mathrm{c}(X)$ определяется равенством $D(A, B)=\max \{\mathrm{e}(A, B), \mathrm{e}(B, A)\}$, где

$$
\mathrm{e}(A, B)=\sup _{x \in A} \operatorname{dist}(x, B) \quad \text { и } \quad \operatorname{dist}(x, B)=\inf _{y \in B} d(x, y), \quad A, B \in \mathrm{c}(X) .
$$

Работа поддержана факультетом математики университета г. Лодзь, Польша (Wydział Matematyki Uniwersytet Łódzki), и Российским фондом фундаментальных исследований, грант № 9901-10644. 
Вопрос о существовании регулярных селекций многозначного отображения ограниченной вариации приводит к отображениям со значениями в таком метрическом пространстве. Ниже изучаются свойства этих отображений: вложения функциональных пространств, структура отображений, скачки функции вариации, принцип выбора Хелли. В теореме 10 показано, что невьпуклозначные и без какого-либо ограничения на график многозначные отображения $F$ из промежутка в $\mathbb{R}$ в компактные подмножества метрического пространства, имеющие конечную обобщенную $\Phi$-вариацию, обладают селекциями, сохраняющими это свойство. Это существенно расширяет результаты о селекциях липшищевых, абсолютно непрерьвных и имеющих ограниченную вариацию отображений, представленные в [7]-[9] ( $X$ конечномерно), а также [10, Д 1.8] и [11]-[15] $(X$ - банахово пространство и $\operatorname{Gr}(F)$ компактен) и [16], [17] (когда $X$ - произвольное метрическое пространство и $\operatorname{Gr}(F)$ произволен). В последней теореме 15 установлено существование (непрерывных) селекций, наследующих свойства компактнозначного многозначного отображения, которое определено на прямом произведении промежутка вещественной прямой и некоторого топологического пространства и имеет конечную обобщенную $\Phi$-вариацию по первому аргументу и полунепрерьвно сверху по второму.

Результаты работы докладывались на XXIII международном летнем симпозиуме по вешественному анализу (21-26 июня 1999 г., г. Лодзь, Польша) и анонсированы в [18].

2. $\varphi$-функции и отображения конечной $\Phi$-вариации. $\Phi$ ункция $\Phi$, действующая из $\mathbb{R}^{+}=[0, \infty)$ в $\mathbb{R}^{+}$, назьвается $\varphi$-функцией (например, $[19, \S 2]$ ), если она является непрерьвной, неубьвающей, неограниченной и $\Phi(\rho)=0$ лишь при $\rho=0$. Будем говорить, что $\varphi$-функция $\Phi$ удовлетворяет условию Орлича или что $\Phi$ являетсл $\varphi$-функиией Орлича, если $\lim _{\rho \rightarrow \infty} \Phi(\rho) / \rho=\infty$. Множество всех выпуклых (вниз) $\varphi$-функций Орлича обозначим через $\mathscr{N}$.

Правая обратная для $\varphi$-функции $\Phi$ определяется правилом [20, гл. $1, \S 2]$

$$
\Phi_{+}^{-1}(r)=\sup \{\rho \geqslant 0 \mid \Phi(\rho) \leqslant r\}, \quad r \in \mathbb{R}^{+} .
$$

$\Phi$ ункция $\Phi_{+}^{-1}$ отображает $\mathbb{R}^{+}$в $\mathbb{R}^{+}$, является непрерывной справа, неубывающей, неограниченной и обрашается в нуль только в нуле, причем справедливы соотношения: $\Phi\left(\Phi_{+}^{-1}(r)\right)=r$ при $r \geqslant 0, \Phi_{+}^{-1}(\Phi(\rho)) \geqslant \rho$ при $\rho \geqslant 0$ и $\Phi_{+}^{-1}(\Phi(\rho)-\varepsilon) \leqslant \rho$ для $\rho>0$ и $0<\varepsilon<\Phi(\rho)$. Функция $\Phi$ есть правая обратная к $\Phi_{+}^{-1}$. Для $\varphi$-функции Орлича $\Phi$ имеем

$$
\lim _{r \rightarrow+0} r \Phi_{+}^{-1}\left(\frac{\vartheta}{r}\right)=\vartheta \lim _{\rho \rightarrow \infty} \frac{\rho}{\Phi(\rho)}=0, \quad \vartheta \in \mathbb{R}^{+}
$$

Любая выпуклая $\varphi$-функция $\Phi$ строго возрастает, поэтому обратная к ней $\Phi^{-1}=\Phi_{+}^{-1}$ непрерьвна и вогнута; кроме того, функции $\rho \mapsto \Phi(\rho) / \rho$ и $\omega_{\Phi}(\rho)=\rho \Phi^{-1}(1 / \rho)$ не убывают при $\rho>0$, поэтому существуют пределы

$$
\lim _{\rho \rightarrow+0} \frac{\Phi(\rho)}{\rho} \in \mathbb{R}^{+}, \quad[\Phi]=\lim _{\rho \rightarrow \infty} \frac{\Phi(\rho)}{\rho} \in(0, \infty] \quad \text { и } \quad \omega_{\Phi}(0)=\lim _{\rho \rightarrow+0} \omega_{\Phi}(\rho) \in \mathbb{R}^{+},
$$

причем $\omega_{\Phi}(0)=0$ тогда и только тогда, когда $\Phi \in \mathcal{N}$.

Пусть $E$ есть бесконечное подмножество $\mathbb{R},(X, d)$ - метрическое пространство и $X^{E_{-}}$ множество всех отображений $f: E \rightarrow X$ из $E$ в $X$. 
Пусть

$$
\mathscr{T}(E)=\left\{T=\left\{t_{i}\right\}_{i=0}^{m} \subset E \mid m \in \mathbb{N}, t_{i-1}<t_{i}, i=1, \ldots, m\right\}
$$

есть множество всех разбиений $E$ конечньми упорядоченными наборами точек из $E$. Для $\varphi$-функции $\Phi, f \in X^{E}$ и $T=\left\{t_{i}\right\}_{i=0}^{m} \in \mathscr{T}(E)$ определим величину

$$
V_{\Phi}[f ; T]=\sum_{i=1}^{m} U\left(t_{i}, t_{i-1}\right), \quad \text { где } \quad U(t, s)=\Phi\left(\frac{d(f(t), f(s))}{t-s}\right)(t-s) .
$$

Отображение $f \in X^{E}$ назьвается отображснием конечной (или ограниченной) $\Phi$-вариачии (в смысле Жордана-Pисса-Орлича), если существует константа $C \geqslant 0$ такая, что $V_{\Phi}[f ; T] \leqslant C$ для всех $T \in \mathscr{T}(E)$. Наименьшая постоянная $C$ с указанньм свойством назьвается (полной) $\Phi$-вариаиией отображения $f$ на $E$ и обозначается через $\mathbf{V}_{\Phi}(f, E)$. Если $E=[a, b]$ есть отрезок, то $\mathscr{T}(E)$ в определении $\mathbf{V}_{\Phi}(f, E)$ можно заменить на множество $\mathscr{T}_{a}^{b}$ всех разбиений $T$ отрезка $[a, b]$, т.е. $T=\left\{t_{i}\right\}_{i=0}^{m}$, где $m \in \mathbb{N}$ и $a=t_{0}<t_{1}<$ $\cdots<t_{m-1}<t_{m}=b$. Множество тех $f \in X^{E}$, для которых $\mathbf{V}_{\Phi}(f, E)<\infty$, обозначим через $B V_{\Phi}(E ; X)$.

Если $\Phi(\rho)=\rho$, то приведенное определение $\mathbf{V}_{\Phi}(f, E)$ дает классическое понятие вариации в смысле Жордана $\left[21\right.$, гл. 8], $[22$, гл. $4, \S 9]$. В этом случае $B V_{\Phi}(E ; X)$ обозначается через $B V_{1}(E ; X)$, а $\mathbf{V}_{\Phi}(f, E)$ - через $\mathbf{V}_{1}(f, E)$ и называется (1-) вариацией отображения $f$ на $E$. Если $\Phi(\rho)=\rho^{q}$, где постоянная $q>1$, то $\mathbf{V}_{\Phi}(f, E)$ определяет понятие $q$-вариации в смысле $\Phi$. Рисса [23, гл. $2, \S 3.36]$. Случай, когда $\Phi$ есть выпуклая $\varphi$-функция Орлича, $E=[a, b]$ - отрезок и $X=\mathbb{R}$, изучался в работах [24]-[26].

Напомним, что если для $f \in X^{E}$ существует постоянная $C \geqslant 0$ такая, что $d(f(t)$, $f(s)) \leqslant C|t-s|$ для всех $t, s \in E$, то $f$ назьвается липиицевыц (запись: $f \in \operatorname{Lip}(E ; X)$ ), а наименьшая такая постоянная $C$ обозначается через $\mathbf{L}(f)$.

Отображение $f \in X^{E}$ называется абсолютно непрерывныцм, если существует функция $\delta:(0, \infty) \rightarrow(0, \infty)$ такая, что для любого $\varepsilon>0$ и любого конечного набора точек $\left\{a_{i}, b_{i}\right\}_{i=1}^{n} \subset E$ таких, что $a_{1}<b_{1} \leqslant a_{2}<b_{2} \leqslant \cdots \leqslant a_{n}<b_{n}$, из условия $\sum_{i=1}^{n}\left(b_{i}-a_{i}\right) \leqslant \delta(\varepsilon)$ вытекает, что $\sum_{i=1}^{n} d\left(f\left(b_{i}\right), f\left(a_{i}\right)\right) \leqslant \varepsilon$. Множество абсолютно непрерьвных отображений из $E$ в $X$ обозначаем через $A C(E ; X)$.

Рассмотрим вкратце случай, когда $(X,\|\cdot\|)$ - банахово пространство. В работе [27] показано, что липшицево отображение $f:[a, b] \rightarrow X$ может оказаться не дифференцируемым ни в одной точке интервала $(a, b)$ ни сильно (в норме $X$ ), ни слабо (на каждом функционале из сильно сопряженного к $X$ пространства), но если $X$ рефлексивно, то любое отображение $f \in A C([a, b] ; X)$ почти всюду на $(a, b)$ сильно дифференцируемо и представимо в виде неопределенного интеграла Бохнера от своей сильной производной $f^{\prime}$. Для вьпуклой $\varphi$-функции $\Phi$ обозначим через $L_{\Phi}^{0}([a, b] ; X)$ пространство всех (классов эквивалентности) сильно измеримых отображений $f \in X^{[a, b]}$, для которых конечен интеграл Лебега $\int_{a}^{b} \Phi(\|f(t)\|) d t$. В работе [14] доказана

Теорема 1. Пусть $X$ - рефлексивное банахово пространство и $\Phi$ - выпуклая $\varphi$-функиия. Любое отображсние $f \in B V_{\Phi}([a, b] ; X)$ почти всюду на $(a, b)$ имеет слабую производную $f^{\bullet} \in L_{\Phi}^{0}([a, b] ; X) u$

$$
\int_{a}^{b} \Phi\left(\left\|f^{\bullet}(t)\right\|\right) d t \leqslant \mathbf{V}_{\Phi}(f,[a, b])
$$


Кроме того, если $\Phi \in \mathcal{N}$, то $f \in B V_{\Phi}([a, b] ; X)$ тогда и только тогда, когда $f \in A C([a, b] ; X)$ и существует сильная производная $f^{\prime} \in L_{\Phi}^{0}([a, b] ; X) ;$ в этом случае имеет место интегральная формула

$$
\mathbf{V}_{\Phi}(f,[a, b])=\int_{a}^{b} \Phi\left(\left\|f^{\prime}(t)\right\|\right) d t
$$

При $X=\mathbb{R}$ критерий в этой теореме известен благодаря $\Phi$. Риссу ([23, гл. $2, \S 3.36]$, когда $\Phi(\rho)=\rho^{q}, q>1$ ), Медведеву [24] и Цибертовичу и Матушевской [25] (если $\Phi \in \mathcal{N})$; в последней работе установлена также интегральная формула для $\Phi$-вариации. Если же $X$ - произвольное метрическое пространство, $\Phi \in \mathscr{N}, f \in X^{[a, b]}$ и $\nu(t)=$ $\mathbf{V}_{1}(f,[a, t]), t \in[a, b]$, то справедлив следующий критерий (установленный в [14] в несколько более общем виде): $f \in B V_{\Phi}([a, b] ; X)$ в том и только том случае, когда $\nu \in$ $A C([a, b] ; \mathbb{R})$ и $\nu^{\prime} \in L_{\Phi}^{0}([a, b] ; \mathbb{R})$. Более того, $\mathbf{V}_{\Phi}(f,[a, b])=\int_{a}^{b} \Phi\left(\left|\nu^{\prime}(t)\right|\right) d t$. В качестве следствия последнего критерия для произвольного метрического пространства $X$ получаем, что

$$
A C([a, b] ; X)=\bigcup_{\Phi \in \mathcal{N}} B V_{\Phi}([a, b] ; X) .
$$

В действительности имеет место более сильное утверждение. Напомним, что (см. [20, гл. $1, \S 5])$ функция $\Phi \in \mathcal{N}$ удовлетворяет $\Delta^{\prime}$-условию, если сушествуют постоянные $C>0$ и $\rho_{0}>0$ такие, что $\Phi\left(\rho_{1} \rho_{2}\right) \leqslant C \Phi\left(\rho_{1}\right) \Phi\left(\rho_{2}\right)$ для всех $\rho_{1} \geqslant \rho_{0}$ и $\rho_{2} \geqslant \rho_{0}$. В силу последнего критерия и [20, гл. $2, \S 8.1]$ имеем: если $X$ - метрическое пространство, то $f \in A C([a, b] ; X)$ тогда и только тогда, когда можно указать удовлетворяющую $\Delta^{\prime}$-условию функцию $\Phi \in \mathcal{N}$ такую, что $f \in B V_{\Phi \circ \Phi}([a, b] ; X) ;$ здесь через $\Phi$ о $\Phi$ обозначена композиция функции $\Phi$ с самой собой.

Всюду ниже (если не сказано обратное) $(X, d)$ - метрическое пространство.

Перечислим основные свойства $\Phi$-вариации, которые потребуются далее. Положим $E_{t}^{-}=E \cap(-\infty, t], E_{t}^{+}=E \cap[t, \infty)$ и $E_{s}^{t}=E \cap[s, t]$, где $t, s \in E, s \leqslant t$.

Лемма 2. Пусть $\Phi$ есть $\varphi$-функиия $u f: E \rightarrow X$. Имеем

(a) ecли $\varnothing \neq A \subset B \subset E$, то $\mathbf{V}_{\Phi}(f, A) \leqslant \mathbf{V}_{\Phi}(f, B)$;

(b) ecлut, $s \in E$ u $s<t, \bmod d(f(t), f(s)) \leqslant(t-s) \Phi_{+}^{-1}\left(\mathbf{V}_{\Phi}(f, E) /(t-s)\right)$;

(c) если $t \in E$, то $\mathbf{V}_{\Phi}\left(f, E_{t}^{-}\right)+\mathbf{V}_{\Phi}\left(f, E_{t}^{+}\right) \leqslant \mathbf{V}_{\Phi}(f, E) ; \kappa$ тому жсе, если $\Phi$ есть выпуклая $\varphi$-функция, то $\mathbf{V}_{\Phi}\left(f, E_{t}^{-}\right)+\mathbf{V}_{\Phi}\left(f, E_{t}^{+}\right)=\mathbf{V}_{\Phi}(f, E)$;

(d) если последовательность $\left\{f_{n}\right\}_{n=1}^{\infty} \subset X^{E} u \lim _{n \rightarrow \infty} d\left(f_{n}(t), f(t)\right)=0$ для всеx $t \in E$, mo $\mathbf{V}_{\Phi}(f, E) \leqslant \liminf _{n \rightarrow \infty} \mathbf{V}_{\Phi}\left(f_{n}, E\right)$;

(e) $\mathbf{V}_{\Phi}(f, E)=\sup \left\{\mathbf{V}_{\Phi}\left(f, E_{s}^{t}\right) \mid s, t \in E, s<t\right\}$;

(f) ecлus $s=\sup E \in \mathbb{R} \cup\{\infty\}$ us $E E, m o \mathbf{V}_{\Phi}(f, E)=\lim _{E \ni t \rightarrow s} \mathbf{V}_{\Phi}\left(f, E_{t}^{-}\right)$;

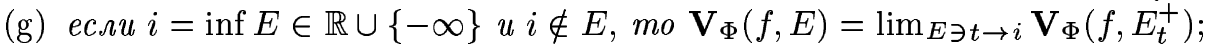

(h) если $s$ и $і$ такие, как в (f) $u(\mathrm{~g}), s \notin E u i \notin E$, то также имеем

$$
\mathbf{V}_{\Phi}(f, E)=\lim _{\substack{E \ni a \rightarrow i \\ E \ni b \rightarrow s}} \mathbf{V}_{\Phi}\left(f, E_{a}^{b}\right)=\lim _{E \ni b \rightarrow s} \lim _{E \ni a \rightarrow i} \mathbf{V}_{\Phi}\left(f, E_{a}^{b}\right)=\lim _{E \ni a \rightarrow i} \lim _{E \ni b \rightarrow s} \mathbf{V}_{\Phi}\left(f, E_{a}^{b}\right)
$$


ДокАЗАТЕЛЬСтво. (а) и (b) являются следствиями определения $Ф$-вариации.

(c) Для разбиений $T_{1} \in \mathscr{T}\left(E_{t}^{-}\right)$и $T_{2} \in \mathscr{T}\left(E_{t}^{+}\right)$положим $\widetilde{T}_{i}=T_{i} \cup\{t\}, i=1,2$. Тогда $\widetilde{T}_{1} \cup \widetilde{T}_{2} \in \mathscr{T}(E)$ и неравенство в (c) вьполняется, поскольку

$$
V_{\Phi}\left[f ; T_{1}\right]+V_{\Phi}\left[f ; T_{2}\right] \leqslant V_{\Phi}\left[f ; \widetilde{T}_{1}\right]+V_{\Phi}\left[f ; \widetilde{T}_{2}\right]=V_{\Phi}\left[f ; \widetilde{T}_{1} \cup \widetilde{T}_{2}\right] \leqslant \mathbf{V}_{\Phi}(f, E) .
$$

Чтобы установить равенство, воспользуемся вьпуклостью функции $\Phi$. Рассмотрим разбиение $T=\left\{t_{i}\right\}_{i=0}^{m} \in \mathscr{T}(E)$. Для случаев, когда $t \in T, t<t_{0}$ или $t_{m}<t$, ясно, что $V_{\Phi}[f ; T] \leqslant \mathbf{V}_{\Phi}\left(f, E_{t}^{-}\right)+\mathbf{V}_{\Phi}\left(f, E_{t}^{+}\right)$. Если теперь $t_{k-1}<t<t_{k}$ при некотором $k \in\{1, \ldots, m\}$, то

$$
V_{\Phi}[f ; T]=\left(\sum_{i=1}^{k-1} U\left(t_{i}, t_{i-1}\right)\right)+U\left(t_{k}, t_{k-1}\right)+\left(\sum_{i=k+1}^{m} U\left(t_{i}, t_{i-1}\right)\right)
$$

(здесь отсутствует первая сумма, если $k=1$, и последняя сумма, если $k=m$ ). Используя неравенство треугольника для $d$, возрастание $\Phi$ и неравенство Йенсена для сумм, находим, что

$$
U\left(t_{k}, t_{k-1}\right) \leqslant U\left(t_{k}, t\right)+U\left(t, t_{k-1}\right),
$$

так что соотношения (4) и (5) дают

$$
V_{\Phi}[f ; T] \leqslant V_{\Phi}\left[f ;\left\{t_{i}\right\}_{i=0}^{k-1} \cup\{t\}\right]+V_{\Phi}\left[f ;\{t\} \cup\left\{t_{i}\right\}_{i=k}^{m}\right] \leqslant \mathbf{V}_{\Phi}\left(f, E_{t}^{-}\right)+\mathbf{V}_{\Phi}\left(f, E_{t}^{+}\right)
$$

(d) Учитывая непрерывность метрики $d$ и функции $\Phi$ и поточечную сходимость $f_{n}$ к $f$, достаточно при любом разбиении $T=\left\{t_{i}\right\}_{i=0}^{m}$ множества $E$ перейти к нижнему пределу при $n \rightarrow \infty$ в неравенстве $V_{\Phi}\left[f_{n} ; T\right] \leqslant \mathbf{V}_{\Phi}\left(f_{n}, E\right)$ и заметить, что

$$
\lim _{n \rightarrow \infty} V_{\Phi}\left[f_{n} ; T\right]=V_{\Phi}[f ; T] \text {. }
$$

(е) В силу пункта (а) левая часть не меньше, чем правая. Обратно, для любого числа $\alpha<\mathbf{V}_{\Phi}(f, E)$ найдется такое разбиение $T=\left\{t_{i}\right\}_{i=0}^{m} \in \mathscr{T}(E)$, что $V_{\Phi}[f ; T] \geqslant \alpha$, но $T \in \mathscr{T}\left(E_{t_{0}}^{t_{m}}\right)$, поэтому $\mathbf{V}_{\Phi}\left(f, E_{t_{0}}^{t_{m}}\right) \geqslant V_{\Phi}[f ; T] \geqslant \alpha$.

(f) Так как $s \notin E$, то $s$ есть предельная точка множества $E$. В силу (а) функция $t \mapsto \mathbf{V}_{\Phi}\left(f, E_{t}^{-}\right)$из $E$ в $[0, \infty]$ не убывает, поэтому написанный в (f) предел существует в $[0, \infty]$ и не превосходит $\mathbf{V}_{\Phi}(f, E)$. С другой стороны, для любого числа $\alpha<\mathbf{V}_{\Phi}(f, E)$ в силу (е) сушествуют числа $a, b \in E, a<b<s$, такие, что $\mathbf{V}_{\Phi}\left(f, E_{a}^{b}\right) \geqslant \alpha$, откуда при любом $t \in E \cap[b, s) \neq \varnothing$ в силу (а) получаем, что $\mathbf{V}_{\Phi}\left(f, E_{t}^{-}\right) \geqslant \mathbf{V}_{\Phi}\left(f, E_{a}^{b}\right) \geqslant \alpha$ и следует равенство в (f).

$(\mathrm{g})$ и первое равенство (h) доказьваются аналогично (f). Для второго имеем

$$
\begin{aligned}
\mathbf{V}_{\Phi}(f, E) & =\lim _{E \ni b \rightarrow s} \mathbf{V}_{\Phi}\left(f, E_{b}^{-}\right)=\lim _{E \ni b \rightarrow s} \lim _{E \ni a \rightarrow i} \mathbf{V}_{\Phi}\left(f,\left(E_{b}^{-}\right)_{a}^{+}\right) \\
& =\lim _{E \ni b \rightarrow s} \lim _{E \ni a \rightarrow i} \mathbf{V}_{\Phi}\left(f, E_{a}^{b}\right) .
\end{aligned}
$$

Последнее равенство в (h) можно доказать точно так же.

Неравенство в лемме $2(\mathrm{~d})$ может быть строгим: последовательность функций

$$
f_{n}(t)=\frac{1}{2 \pi n} \cos (2 \pi n t)
$$

равномерно относительно $t \in[0,1]$ сходится при $n \rightarrow \infty$ к функции $f \equiv 0$, а интегральная формула для $\Phi$-вариации дает

$$
\mathbf{V}_{\Phi}\left(f_{n},[0,1]\right)=\frac{2}{\pi} \int_{0}^{\pi / 2} \Phi(\sin t) d t>0
$$

где $n \in \mathbb{N}$ и $\Phi$ - выпуклая $\varphi$-функция. 


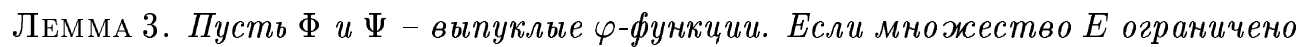
и выполняется условие $\Delta_{\Psi}^{\Phi}$ :

$$
\limsup _{\rho \rightarrow \infty} \frac{\Psi(\rho)}{\Phi(\rho)}<\infty
$$

то $B V_{\Phi}(E ; X) \subset B V_{\Psi}(E ; X)$. Обратно, если $E=[a, b]-$ отрезок, $(X,\|\cdot\|)-л и-$ нейное нормированное пространство и $B V_{\Phi}(E ; X) \subset B V_{\Psi}(E ; X)$, то имеет место условие $\Delta_{\Psi}^{\Phi}$.

ДокАЗАТЕльство. Условие $\Delta_{\Psi}^{\Phi}$ эквивалентно тому, что $\Psi(\rho) \leqslant C \Phi(\rho)$ для всех $\rho \geqslant \rho_{0}$, где $C>0$ и $\rho_{0}>0$ - некоторые постоянные. Для $f \in B V_{\Phi}(E ; X)$ и $T \in \mathscr{T}(E)$ тог да имеем $V_{\Psi}[f ; T] \leqslant \Psi\left(\rho_{0}\right)(\sup E-\inf E)+C \mathbf{V}_{\Phi}(f, E)$.

Напомним, что вьпуклая $\varphi$-функция $\Phi$ имеет неубьвающую непрерьвную справа правую производную $\Phi_{+}^{\prime}(\rho), \rho \geqslant 0$, причем $\Phi_{+}^{\prime}(\rho)>0$ при $\rho>0$, поэтому

$$
\exists \rho(\Phi)>0 \quad \mathrm{c}(\Phi)>0: \quad \forall \rho \geqslant \rho(\Phi) \quad \Phi(\rho) \geqslant \mathrm{c}(\Phi) \rho .
$$

Если теперь условие $\Delta_{\Psi}^{\Phi}$ нарушается, то найдется возрастающая к бесконечности последовательность положительных чисел $\left\{\rho_{n}\right\}_{n=1}^{\infty}$, для которой $\Psi\left(\rho_{n}\right)>2^{n} \Phi\left(\rho_{n}\right)$, $n \in \mathbb{N}$. Определим последовательность $\left\{a_{n}\right\}_{n=0}^{\infty}$ в $[a, b]$ рекуррентно:

$$
a_{0}=a, \quad a_{n}-a_{n-1}=2^{-n}(b-a) \frac{\Phi\left(\rho_{1}\right)}{\Phi\left(\rho_{n}\right)}, \quad n \in \mathbb{N} .
$$

Положим

$$
f(t)= \begin{cases}\left(\rho_{n}\left(t-a_{n-1}\right)+S_{n-1}\right) x, & \text { если } a_{n-1} \leqslant t<a_{n}, n \in \mathbb{N} \\ S_{\infty} x, & \text { если } \lim _{n \rightarrow \infty} a_{n} \leqslant t \leqslant b\end{cases}
$$

где

$$
S_{0}=0, \quad S_{k}=\sum_{i=1}^{k} \rho_{i}\left(a_{i}-a_{i-1}\right), \quad k \in \mathbb{N} \cup\{\infty\},
$$

$S_{\infty}<\infty$ в силу (6), $x \in X,\|x\|=1$. Покажем, что $f \in B V_{\Phi}([a, b] ; X)$, но $f \notin B V_{\Psi}([a, b]$; $X)$. Действительно,

$$
\mathbf{V}_{\Phi}(f,[a, b])=\sum_{n=1}^{\infty} U\left(a_{n}, a_{n-1}\right)=\sum_{n=1}^{\infty} \Phi\left(\rho_{n}\right)\left(a_{n}-a_{n-1}\right)=(b-a) \Phi\left(\rho_{1}\right)<\infty
$$

С другой стороны, для произвольного $m \in \mathbb{N}$ и $T_{m}=\left\{a_{n}\right\}_{n=0}^{m}$ имеем

$$
\mathbf{V}_{\Psi}(f,[a, b]) \geqslant V_{\Psi}\left[f ; T_{m}\right]=\sum_{n=1}^{m} \Psi\left(\rho_{n}\right) \frac{(b-a) \Phi\left(\rho_{1}\right)}{2^{n} \Phi\left(\rho_{n}\right)} \geqslant m(b-a) \Phi\left(\rho_{1}\right) .
$$

Из леммы 3 и утверждения (6) для вьпуклой $\varphi$-функции $\Phi$ вытекает вложение множества $B V_{\Phi}(E ; X)$ в $B V_{1}(E ; X)$, а кроме того, если величина [ $\left.\Phi\right]$ (равная также супремуму $\left.\sup _{\rho>0} \Phi(\rho) / \rho\right)$ конечна, то $B V_{\Phi}(E ; X)=B V_{1}(E ; X)$, что следует из неравенства $\mathbf{V}_{\Phi}(f, E) \leqslant[\Phi] \mathbf{V}_{1}(f, E)$ для $f \in B V_{1}(E ; X)$. 
3. Пространство отображений обобщенной $\Phi$-вариации. Пусть $(X, d)$ - метрическое пространство и $E \subset \mathbb{R}$. Для $\lambda>0$ и вьпуклой $\varphi$-функции $\Phi$ положим $\Phi_{\lambda}(\rho)=$ $\Phi(\rho / \lambda), \rho \in \mathbb{R}^{+}$. Там, где это не вызывает недоразумений, для краткости пишем $B V_{\Phi}$ вместо $B V_{\Phi}(E ; X)$. Из леммы 3 находим, что $B V_{\Phi_{\lambda}} \subset B V_{\Phi}$ при $0<\lambda \leqslant 1$ и $B V_{\Phi} \subset B V_{\Phi_{\lambda}}$ при $\lambda>1$. Последнее включение, вообще говоря, строгое: например, если $\Phi(\rho)=e^{\rho}-1$, $f(t)=t(1-\log t)$ при $0<t \leqslant 1$ и $f(0)=0$, то, используя интегральную формулу для $\Phi$-вариации из теоремы 1 , получим $\mathbf{V}_{\Phi_{\lambda}}(f,[0,1])=1 /(\lambda-1)$ при $\lambda>1$ и $\mathbf{V}_{\Phi_{\lambda}}(f,[0,1])=\infty$ при $0<\lambda \leqslant 1$. В силу леммы 3 обратное включение $B V_{\Phi_{\lambda}} \subset B V_{\Phi}$ при $\lambda>1$ имеет место тогда и только тогда, когда $\Phi$ удовлетворяет $\Delta_{2}$-условию (см. [20, гл. $1, \S 4],[19, \S 3]): \lim _{\sup } \rightarrow \infty \Phi(2 \rho) / \Phi(\rho)<\infty$, а это условие эквивалентно следующему: для любого числа $\lambda>1$ существуют постоянные $C(\lambda)>0$ и $\rho_{0}(\lambda)>0$ такие, что $\Phi(\rho) \leqslant C(\lambda) \Phi(\rho / \lambda)$ для всех $\rho \geqslant \rho_{0}(\lambda)$. (Отметим, что если $[\Phi]<\infty$, то $\Phi$ удовлетворяет $\Delta_{2}$-условию.)

В общем случае для любой вьпуклой $\varphi$-функции $\Phi$ пространство отображсений конечной обобщенной $\Phi$-вариаиии - это множество

$$
G V_{\Phi}(E ; X)=\bigcup_{\lambda>0} B V_{\Phi_{\lambda}}(E ; X)
$$

Из сказанного выше следует, что если выпуклая $\varphi$-функция $\Phi$ удовлетворяет $\Delta_{2}$-условию, то $G V_{\Phi}=B V_{\Phi}$. Обратно, если $E=[a, b], X$ - линейное нормированное пространство и $G V_{\Phi}=B V_{\Phi}$, то $\Phi$ удовлетворяет $\Delta_{2}$-условию: в силу леммы 3 и вложения $B V_{\Phi_{2}} \subset B V_{\Phi}$ найдутся $C>0$ и $\rho_{0}>0$ такие, что $\Phi(\rho) \leqslant C \Phi(\rho / 2), \rho \geqslant \rho_{0}$.

Если $\Phi$ и $\Psi$ - вьпуклые $\varphi$-функции, множество $E$ ограничено и найдутся такие постоянные $C>0$ и $\rho_{0}>0$, что $\Psi(\rho) \leqslant \Phi(C \rho)$ при $\rho \geqslant \rho_{0}$, то $G V_{\Phi} \subset G V_{\Psi}$, так как для $\lambda>0, f \in B V_{\Phi_{\lambda}}$ и $T \in \mathscr{T}(E)$ имеем $V_{\Psi_{\mu}}[f ; T] \leqslant \Psi\left(\rho_{0}\right)|\sup E-\inf E|+\mathbf{V}_{\Phi_{\lambda}}(f, E)$, где $\mu=\lambda C$. Обратное утверждение для $E=[a, b]$ и линейного нормированного пространства $X$ установлено в [15].

Для рефлексивного банахова пространства $X$ и $\Phi \in \mathscr{N}$ из теоремы 1 имеем $f \in$ $G V_{\Phi}([a, b] ; X)$ тогда и только тогда, когда $f \in A C([a, b] ; X)$ и $f^{\prime} \in L_{\Phi}([a, b] ; X)$, где через $L_{\Phi}([a, b] ; X)$ обозначено пространство Орлича [20], [19] тех отображений $f \in X^{[a, b]}$, что $\lambda f \in L_{\Phi}^{0}([a, b] ; X)$ при некотором $\lambda>0$.

В силу выпуклости функции $\Phi$ корректно определена величина

$$
p_{\Phi}(f, E)=\inf \left\{\lambda>0 \mid \mathbf{V}_{\Phi_{\lambda}}(f, E) \leqslant 1\right\}, \quad f \in G V_{\Phi}(E ; X)
$$

основные свойства которой отражены в следующей лемме.

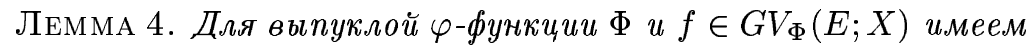

(a) $d(f(t), f(s)) \leqslant \omega_{\Phi}(|t-s|) p_{\Phi}(f, E)$ для всех $t, s \in E$;

(b) если $p_{\Phi}(f, E)=\lambda>0$, mo $\mathbf{V}_{\Phi_{\lambda}}(f, E) \leqslant 1$;

(c) если $\lambda>0$, то $p_{\Phi}(f, E) \leqslant \lambda$ тогда и только тогда, когда $\mathbf{V}_{\Phi_{\lambda}}(f, E) \leqslant 1$;

(d) ecли $\lambda>0 u \mathbf{V}_{\Phi_{\lambda}}(f, E)=1$, mo $p_{\Phi}(f, E)=\lambda$;

(e) ecли $\left\{f_{n}\right\}_{n=1}^{\infty} \subset G V_{\Phi}(E ; X) u \lim _{n \rightarrow \infty} d\left(f_{n}(t), f(t)\right)=0$ для всех $t \in E$, то $p_{\Phi}(f, E) \leqslant \liminf _{n \rightarrow \infty} p_{\Phi}\left(f_{n}, E\right)$

(f) $e c л u t \in E, \operatorname{mo} p_{\Phi}(f, E) \leqslant p_{\Phi}\left(f, E_{t}^{-}\right)+p_{\Phi}\left(f, E_{t}^{+}\right)$. 
ДокаЗАТЕЛьСтво. (а) Для любых $t, s \in E, s<t$, из определений имеем

$$
\Phi\left(\frac{d(f(t), f(s))}{(t-s) \lambda}\right)(t-s) \leqslant \mathbf{V}_{\Phi_{\lambda}}(f, E) \leqslant 1 \quad \text { при } \lambda>p_{\Phi}(f),
$$

откуда, разделив на $t-s$ и взяв обратную функцию $\Phi^{-1}$, придем к (a).

(b) Пусть $p_{\Phi}(f)=\lambda>0$. Выберем числа $\lambda(n)>\lambda, n \in \mathbb{N}$, такие, что

$$
\lim _{n \rightarrow \infty} \lambda(n)=\lambda
$$

Поскольку $\mathbf{V}_{\Phi_{\lambda(n)}}(f, E) \leqslant 1$ для всех $n \in \mathbb{N}$, находим, что

$$
\mathbf{V}_{\Phi_{\lambda}}(f, E)=\lim _{n \rightarrow \infty} \mathbf{V}_{\Phi_{\lambda(n)}}(f, E) \leqslant 1
$$

(c) Достаточно показать, что если $0<p_{\Phi}(f)<\lambda$, то $\mathbf{V}_{\Phi_{\lambda}}(f, E)<1$, а это сразу вытекает из вьпуклости $\Phi$ и (b): полагая $\mu=p_{\Phi}(f)$, имеем

$$
\mathbf{V}_{\Phi_{\lambda}}(f, E) \leqslant(\mu / \lambda) \mathbf{V}_{\Phi_{\mu}}(f, E) \leqslant \mu / \lambda<1
$$

(d) Следует только заметить, что ввиду (c) и только что доказанного утверждения случаи, когда $p_{\Phi}(f)>\lambda$ и $p_{\Phi}(f)<\lambda$, невозможны.

(е) Предположим, что $\lambda=\liminf _{n \rightarrow \infty} p_{\Phi}\left(f_{n}\right)<\infty$. Тогда $p_{\Phi}\left(f_{n_{k}}\right) \rightarrow \lambda$ при $k \rightarrow \infty$ для некоторой подпоследовательности $\left\{f_{n_{k}}\right\}_{k=1}^{\infty}$ в $\left\{f_{n}\right\}_{n=1}^{\infty}$, поэтому для любого $\varepsilon>0$ найдется такое $k_{0}(\varepsilon) \in \mathbb{N}$, что $p_{\Phi}\left(f_{n_{k}}\right)<\lambda+\varepsilon$ для всех $k \geqslant k_{0}(\varepsilon)$. Из определения $p_{\Phi}\left(f_{n_{k}}\right)$ следует, что $\mathbf{V}_{\Phi_{\lambda+\varepsilon}}\left(f_{n_{k}}\right) \leqslant 1$ при $k \geqslant k_{0}(\varepsilon)$, а поскольку $f_{n_{k}}$ сходится к $f$ поточечно на $E$ при $k \rightarrow \infty$, то лемма $2(\mathrm{~d})$ дает $\mathbf{V}_{\Phi_{\lambda+\varepsilon}}(f) \leqslant \lim \inf _{k \rightarrow \infty} \mathbf{V}_{\Phi_{\lambda+\varepsilon}}\left(f_{n_{k}}\right) \leqslant 1$, откуда $p_{\Phi}(f) \leqslant \lambda+\varepsilon$ для любого $\varepsilon>0$.

(f) Положим $\lambda=p_{\Phi}\left(f, E_{t}^{-}\right)$и $\mu=p_{\Phi}\left(f, E_{t}^{+}\right)$. Если хотя бы одно из чисел $\lambda$ или $\mu$ равно нулю, то в силу (а) неравенство (в действительности равенство) очевидно. Пусть $\lambda>0$ и $\mu>0$. Из (b) получаем, что $\mathbf{V}_{\Phi_{\lambda}}\left(f, E_{t}^{-}\right) \leqslant 1$ и $\mathbf{V}_{\Phi_{\mu}}\left(f, E_{t}^{+}\right) \leqslant 1$. Неравенство $p_{\Phi}(f, E) \leqslant \lambda+\mu$ в силу (c) эквивалентно неравенству $\mathbf{V}_{\Phi_{\lambda+\mu}}(f, E) \leqslant 1$. Чтобы доказать последнее, рассмотрим разбиение $T=\left\{t_{i}\right\}_{i=0}^{m}$ множества $E$ такое, что $t_{k-1} \leqslant t \leqslant t_{k}$ при некотором $k \in\{1, \ldots, m\}$ (случаи, когда $t<t_{0}$ и $t>t_{m}$, разбираются аналогично). Обозначим через $U_{\lambda}(t, s)$ выражение $U(t, s)$ в $(3)$, соответствующее функции $\Phi_{\lambda}$. Для величины $V_{\Phi_{\lambda+\mu}}[f ; T]$ из (3) имеем равенство (4), где $U$ заменено на $U_{\lambda+\mu}$. С учетом выпуклости функции $\Phi$ справедливы неравенства

$$
\begin{gathered}
U_{\lambda+\mu}\left(t_{i}, t_{i-1}\right) \leqslant \frac{\lambda}{\lambda+\mu} U_{\lambda}\left(t_{i}, t_{i-1}\right), \quad i=1, \ldots, k-1, \\
U_{\lambda+\mu}\left(t_{k}, t_{k-1}\right) \leqslant U_{\lambda+\mu}\left(t, t_{k-1}\right)+U_{\lambda+\mu}\left(t_{k}, t\right) \leqslant \frac{\lambda}{\lambda+\mu} U_{\lambda}\left(t, t_{k-1}\right)+\frac{\mu}{\lambda+\mu} U_{\mu}\left(t_{k}, t\right), \\
U_{\lambda+\mu}\left(t_{i}, t_{i-1}\right) \leqslant \frac{\mu}{\lambda+\mu} U_{\mu}\left(t_{i}, t_{i-1}\right), \quad i=k+1, \ldots, m,
\end{gathered}
$$

которые вместе с (4) дают

$$
V_{\Phi_{\lambda+\mu}}[f ; T] \leqslant \frac{\lambda}{\lambda+\mu} \mathbf{V}_{\Phi_{\lambda}}\left(f, E_{t}^{-}\right)+\frac{\mu}{\lambda+\mu} \mathbf{V}_{\Phi_{\mu}}\left(f, E_{t}^{+}\right) \leqslant 1 .
$$


Одним из преимуществ перехода к пространству $G V_{\Phi}(E ; X)$ является то, что понятие отображения $f \in G V_{\Phi}(E ; X)$ конечной обобщенной $\Phi$-вариации зависит от метрической топологии на $X$, а не от конкретной метрики: действительно, если $d$ и $d_{0}$ - эквивалентные метрики на $X$, т.е. $C_{0} d(x, y) \leqslant d_{0}(x, y) \leqslant C_{1} d(x, y)$ для некоторых постоянных $C_{0}>0$ и $C_{1}>0$ и всех $x, y \in X$, и $f \in G V_{\Phi}(E ; X)$ относительно метрики $d$, то $f \in G V_{\Phi}(E ; X)$ относительно метрики $d_{0}$ и справедливы неравенства

$$
C_{0} p_{\Phi}^{d}(f, E) \leqslant p_{\Phi}^{d_{0}}(f, E) \leqslant C_{1} p_{\Phi}^{d}(f, E)
$$

где через $p_{\Phi}^{d}(f, E)$ обозначена величина (8), вычисленная в метрике $d$.

Суммируя сказанное вьше, для ограниченного множества $E$ и выпуклой $\varphi$-функции $\Phi$ имеем следующие вложения функциональных пространств:

$$
\operatorname{Lip}(E ; X) \subset B V_{\Phi}(E ; X) \subset G V_{\Phi}(E ; X) \subset B V_{1}(E ; X)
$$

а если, дополнительно, $\Phi \in \mathcal{N}$, то $G V_{\Phi}(E ; X) \subset A C(E ; X)$. (Отметим, что если $\Phi$ есть не обязательно вьпуклая $\varphi$-функция Орлича, то в силу леммы $4($ а) и (2) любое отображение $f \in G V_{\Phi}(E ; X)$ является непрерьвньм.) Кроме того, имеют место неравенства Йенсена для вариачии:

$$
\begin{array}{rlrl}
\Phi\left(\frac{\mathbf{V}_{1}(f, E)}{|E|}\right) & \leqslant \frac{\mathbf{V}_{\Phi}(f, E)}{|E|}, & & f \in B V_{\Phi}(E ; X) \\
\mathbf{V}_{1}(f, E) & \leqslant \omega_{\Phi}(|E|) p_{\Phi}(f, E), & f \in G V_{\Phi}(E ; X)
\end{array}
$$

где $|E|=\sup E-\inf E<\infty$. Действительно, если $f \in B V_{\Phi}(E ; X)$ и $T$ есть разбиение $E$ вида $\left\{t_{i}\right\}_{i=0}^{m}$, то, используя обозначение $U$ из $(3)$, в силу неравенства Йенсена для сумм имеем

$$
\Phi\left(\frac{\sum_{i=1}^{m} d\left(f\left(t_{i}\right), f\left(t_{i-1}\right)\right)}{\sum_{i=1}^{m}\left(t_{i}-t_{i-1}\right)}\right) \leqslant \frac{\sum_{i=1}^{m} U\left(t_{i}, t_{i-1}\right)}{\sum_{i=1}^{m}\left(t_{i}-t_{i-1}\right)} \leqslant \frac{\mathbf{V}_{\Phi}(f, E)}{\sum_{i=1}^{m}\left(t_{i}-t_{i-1}\right)},
$$

откуда, учитьвая, что $\sum_{i=1}^{m}\left(t_{i}-t_{i-1}\right)=t_{m}-t_{0} \leqslant|E|$, придем к $(10)$. Если же $f \in G V_{\Phi}(E ; X)$, то, полагая $\lambda=\mathbf{V}_{1}(f, E) / \omega_{\Phi}(|E|)$ и считая, что $\lambda>0$, в силу (10) имеем $\mathbf{V}_{\Phi_{\lambda}}(f, E) \geqslant|E| \Phi_{\lambda}\left(\mathbf{V}_{1}(f, E) /|E|\right)=1$, поэтому из утверждения в доказательстве леммы $4(\mathrm{c})$ получаем, что $p_{\Phi}(f, E) \geqslant \lambda$.

Структурно отображения конечной $\Phi$-вариации тесно связаны с липшицевьги отображениями и вещественными функциями конечной (обобщенной) $\Phi$-вариации. Пусть функция $\nu: E \rightarrow \mathbb{R}, J=\nu(E)$ есть образ $\nu, g \in \operatorname{Lip}(J ; X), \mathbf{L}(g) \leqslant 1$ и $f=g \circ \nu$, где $(g \circ \nu)(t):=g(\nu(t)), t \in E$. Для выпуклой $\varphi$-функции $\Phi$ справедливы утверждения: если $\nu \in B V_{\Phi}(E ; \mathbb{R})$, то $f \in B V_{\Phi}(E ; X)$ и $\mathbf{V}_{\Phi}(f, E) \leqslant \mathbf{V}_{\Phi}(\nu, E) ;$ если $\nu \in G V_{\Phi}(E ; \mathbb{R})$, то $f \in G V_{\Phi}(E ; X)$ и $p_{\Phi}(f, E) \leqslant p_{\Phi}(\nu, E)$. В лемме 5 мы покажем, что справедливы и обратные утверждения.

Отображение $g: J \rightarrow X$ называется натуральным, если $\mathbf{V}_{1}\left(g, J_{s}^{t}\right)=t-s$ для всех $s, t \in J, s \leqslant t$. Такое отображение липшицево и $\mathbf{L}(g) \leqslant 1$, поскольку $d(g(t), g(s)) \leqslant$ $\mathbf{V}_{1}\left(g, J_{s}^{t}\right)$ в силу леммы $2(\mathrm{~b})$ при $\Phi(\rho)=\rho$. 
Лемма 5. Пусть $f \in B V_{1}(E ; X), \nu(t)=\mathbf{V}_{1}\left(f, E_{t}^{-}\right), t \in E, u J=\nu(E)$. Тогда существует такое натуральное отображение $g: J \rightarrow X$, что $f=g \circ \nu$ на $E u$ $\mathbf{V}_{1}(g, J)=\mathbf{V}_{1}(f, E)$. Если Е ограничено $и \Phi-$ выпуклая $\varphi$-функиия, дополнительно имеем следующие утвер ждения:

(a) если $f \in B V_{\Phi}(E ; X)$, то $\nu \in B V_{\Phi}(E ; \mathbb{R}) u \mathbf{V}_{\Phi}(\nu, E)=\mathbf{V}_{\Phi}(f, E)$;

(b) если $f \in G V_{\Phi}(E ; X)$, то $\nu \in G V_{\Phi}(E ; \mathbb{R}) u p_{\Phi}(\nu, E)=p_{\Phi}(f, E)$.

ДокАЗАТЕЛЬСТво. В силу (9) и леммы $2(\mathrm{a})$ функция $\nu$ корректно определена и не убывает на $E$. Если $s \in J$, так что $s=\nu(t)$ для некоторого $t \in E$, то положим $g(s)=f(t)$. Поскольку $d(f(t), f(s)) \leqslant|\nu(t)-\nu(s)|, t, s \in E$, отображение $g: J \rightarrow X$ определено корректно и удовлетворяет всем условиям нашей леммы (подробнее см. [12, $\S 3])$. В условиях (а) для разбиения $T=\left\{t_{i}\right\}_{i=0}^{m} \in \mathscr{T}(E)$ из леммы $2(\mathrm{c})$ при $\Phi(\rho)=\rho$ и неравенства (10) для $i=1, \ldots, m$ находим, что

$$
\nu\left(t_{i}\right)-\nu\left(t_{i-1}\right)=\mathbf{V}_{1}\left(f, E_{t_{i-1}}^{t_{i}}\right) \leqslant\left(t_{i}-t_{i-1}\right) \Phi^{-1}\left(\frac{\mathbf{V}_{\Phi}\left(f, E_{t_{i-1}}^{t_{i}}\right)}{t_{i}-t_{i-1}}\right),
$$

откуда, вычисляя $V_{\Phi}[\nu ; T]$ по формуле (3), снова при помощи леммы $2($ с) получим неравенство $V_{\Phi}[\nu ; T] \leqslant \mathbf{V}_{\Phi}(f, E)$. Равенство же $\mathbf{V}_{\Phi}(\nu, E)=\mathbf{V}_{\Phi}(f, E)$ вытекает из разложения $f=g \circ \nu$ и замечаний перед этой леммой.

Если $f \in G V_{\Phi}(E ; X)$, то, полагая $\lambda=p_{\Phi}(f, E)$ (пусть $\left.\lambda>0\right)$, в силу доказанного в (а) и леммы $4(\mathrm{~b})$ найдем, что $\mathbf{V}_{\Phi_{\lambda}}(\nu, E)=\mathbf{V}_{\Phi_{\lambda}}(f, E) \leqslant 1$, откуда $p_{\Phi}(\nu, E) \leqslant \lambda$. Обратное неравенство следует из замечаний перед леммой.

4. Скачки функции $\Phi$-вариации и непрерывность. Для $t \in E$ обозначим через $E_{t}^{-\prime}$ и $E_{t}^{+\prime}$ множества всех предельных точек для $E_{t}^{-}$и $E_{t}^{+}$соответственно. Встречающиеся ниже пределы вида $\lim _{E \ni \alpha \rightarrow t \pm 0}$ обозначаются короче $\lim _{\alpha \rightarrow t \pm 0}$. В этом пункте $\Phi$ есть выпуклая $\varphi$-функция такая, что $[\Phi]<\infty, f \in B V_{\Phi}(E ; X)$ и $\phi(t)=\mathbf{V}_{\Phi}\left(f, E_{t}^{-}\right)$, $t \in E$, есть функиия $\Phi$-вариации отображения $f$. Мы изучим свойства непрерьвности $f$; покажем, что точки разрьва $f$ совпадают с точками разрьва $\phi$; установим связь между скачками $f$ и $\phi$ и получим формулы для $\Phi$-вариации $f$ на множестве $E$ без удаленной из него предельной точки.

Если $U$ определено в (3), то, как уже отмечалось выше (см. (5) и лемму $2(\mathrm{~b}, \mathrm{c}))$, для всех $\alpha, \beta, \gamma \in E, \alpha<\beta<\gamma$, имеют место неравенства

$$
U(\gamma, \alpha) \leqslant U(\gamma, \beta)+U(\beta, \alpha), \quad U(\beta, \alpha) \leqslant \phi(\beta)-\phi(\alpha) .
$$

Из монотонности и ограниченности функции $\phi$ вытекает, что существуют конечные пределы справа и слева $\phi(t \pm 0)=\lim _{\alpha \rightarrow t \pm 0} \phi(\alpha)$ в точках $t \in E_{t}^{ \pm \prime}$ соответственно. Поскольку этим же свойством обладает функция $\nu$ из леммы 5, из (3) следует, что конечны следуюшие (односторонние) пределы:

$$
\begin{array}{rll}
U(t, t-0) & =\lim _{\alpha \rightarrow t-0} U(t, \alpha)=[\Phi] \lim _{\alpha \rightarrow t-0} d(f(t), f(\alpha)), \quad t \in E_{t}^{-\prime}, \\
U(t+0, t) & =\lim _{\beta \rightarrow t+0} U(\beta, t)=[\Phi] \lim _{\beta \rightarrow t+0} d(f(\beta), f(t)), \quad t \in E_{t}^{+\prime}, \\
U(t+0, t-0) & =\lim _{\substack{\alpha \rightarrow t-0 \\
\beta \rightarrow t+0}} U(\beta, \alpha)=[\Phi] \lim _{\substack{\alpha \rightarrow t-0 \\
\beta \rightarrow t+0}} d(f(\beta), f(\alpha)), \quad t \in E_{t}^{-\prime} \cap E_{t}^{-\prime} .
\end{array}
$$


Теорема 6. Имеют место следующие равенства:

(a) $U(t, t-0)=\phi(t)-\phi(t-0)=\mathbf{V}_{\Phi}\left(f, E_{t}^{-}\right)-\mathbf{V}_{\Phi}\left(f, E_{t}^{-} \backslash\{t\}\right)=\lim _{\alpha \rightarrow t-0} \mathbf{V}_{\Phi}\left(f, E_{\alpha}^{t}\right)$;

(b) $U(t+0, t)=\phi(t+0)-\phi(t)=\mathbf{V}_{\Phi}\left(f, E_{t}^{+}\right)-\mathbf{V}_{\Phi}\left(f, E_{t}^{+} \backslash\{t\}\right)=\lim _{\beta \rightarrow t+0} \mathbf{V}_{\Phi}\left(f, E_{t}^{\beta}\right)$;

(c) $U(t+0, t-0)=\lim _{\substack{\alpha \rightarrow t-0 \\ \beta \rightarrow t+0}} \mathbf{V}_{\Phi}\left(f, E_{\alpha}^{\beta} \backslash\{t\}\right)$;

(d) $\mathbf{V}_{\Phi}(f, E)=\mathbf{V}_{\Phi}(f, E \backslash\{t\})+\phi(t+0)-\phi(t-0)-U(t+0, t-0)$, əде: $\quad \boldsymbol{\theta}$ (a) точка $t \in E_{t}^{-\prime} ; \boldsymbol{\theta}$ (b) точка $t \in E_{t}^{+\prime} ; \boldsymbol{\theta}(\mathrm{c})$, (d) точка $t \in E_{t}^{-\prime} \cap E_{t}^{+\prime}$.

ДокАЗАТЕЛьство. (а) Установим первое равенство. Переходя к пределу $\alpha \rightarrow t-0$ во втором неравенстве (12), где $\beta=t>\alpha \in E$, найдем, что $U(t, t-0) \leqslant \phi(t)-\phi(t-0)$. Обратное неравенство вытекает в пределе при $\alpha \rightarrow t-0$ из следующего утверждения: для любого $\varepsilon>0$ существует $\tau(\varepsilon) \in E, \tau(\varepsilon)<t$, такое, что $\phi(t)-\phi(\alpha) \leqslant U(t, \alpha)+\varepsilon$ для всех $\alpha \in E_{\tau(\varepsilon)}^{t} \backslash\{t\}$. Из определения $\phi(t)$ по $\varepsilon>0$ найдем зависящее от $\varepsilon$ разбиение $T=\left\{t_{i}\right\}_{i=0}^{m} \cup\{t\} \in \mathscr{T}\left(E_{t}^{-}\right)$, где $t_{m}<t$, для которого $\phi(t) \leqslant U\left(t, t_{m}\right)+V_{\Phi}[f ; T]+\varepsilon$. Поскольку $T \in \mathscr{T}\left(E_{t_{m}}^{-}\right)$, применяя первое неравенство в $(12)$ и лемму $2(\mathrm{~b}, \mathrm{c})$, для всех $\alpha \in E, t_{m}<\alpha<t$, получим

$$
\phi(t) \leqslant U(t, \alpha)+\mathbf{V}_{\Phi}\left(f, E_{t_{m}}^{\alpha}\right)+\mathbf{V}_{\Phi}\left(f, E_{t_{m}}^{-}\right)+\varepsilon=U(t, \alpha)+\phi(\alpha)+\varepsilon
$$

и остается положить $\tau(\varepsilon)=t_{m}$.

Оставшиеся равенства вытекают из леммы $2(\mathrm{f})$, в силу которой

$$
\mathbf{V}_{\Phi}\left(f, E_{t}^{-} \backslash\{t\}\right)=\lim _{\alpha \rightarrow t-0} \mathbf{V}_{\Phi}\left(f,\left(E_{t}^{-} \backslash\{t\}\right)_{\alpha}^{-}\right)=\lim _{\alpha \rightarrow t-0} \mathbf{V}_{\Phi}\left(f, E_{\alpha}^{-}\right)=\phi(t-0) .
$$

(b) Неравенство $U(t+0, t) \leqslant \phi(t+0)-\phi(t)$ получается в пределепри $\beta \rightarrow t+0$ из второго неравенства (12), где $E \ni \beta>t=\alpha$. Остается показать, что для любого $\varepsilon>0$ существует $t_{0}=t_{0}(\varepsilon) \in E, t_{0}>t$, такое, что $\phi(\beta)-\phi(t) \leqslant U(\beta, t)+\varepsilon$ для всех $\beta \in E_{t}^{t_{0}} \backslash\{t\}$. Так как $\mathbf{V}_{\Phi}\left(f, E_{t}^{+}\right)<\infty$, то для $\varepsilon>0$ существует $T=\left\{t_{i}\right\}_{i=0}^{m} \cup\{t\} \in \mathscr{T}\left(E_{t}^{+}\right)$c $t_{0}>t$ такое, что $\mathbf{V}_{\Phi}\left(f, E_{t}^{+}\right) \leqslant U\left(t_{0}, t\right)+V_{\Phi}[f ; T]+\varepsilon$, а поскольку $T \in \mathscr{T}\left(E_{t_{0}}^{+}\right)$, то для $\beta \in E$, $t<\beta<t_{0}$, в силу первого неравенства (12) найдем, что

$$
\mathbf{V}_{\Phi}\left(f, E_{t}^{+}\right) \leqslant \mathbf{V}_{\Phi}\left(f, E_{\beta}^{t_{0}}\right)+U(\beta, t)+\mathbf{V}_{\Phi}\left(f, E_{t_{0}}^{+}\right)+\varepsilon=\mathbf{V}_{\Phi}\left(f, E_{\beta}^{+}\right)+U(\beta, t)+\varepsilon,
$$

откуда в силу леммы 2(c) получим

$$
\phi(\beta)-\phi(t)=\mathbf{V}_{\Phi}\left(f, E_{\beta}^{-}\right)-\mathbf{V}_{\Phi}\left(f, E_{t}^{-}\right)=\mathbf{V}_{\Phi}\left(f, E_{t}^{+}\right)-\mathbf{V}_{\Phi}\left(f, E_{\beta}^{+}\right) \leqslant U(\beta, t)+\varepsilon .
$$

Второе и третье равенства следуют из леммы 2 (g,c), в силу чего

$$
\begin{aligned}
\mathbf{V}_{\Phi}\left(f, E_{t}^{+} \backslash\{t\}\right) & =\lim _{\beta \rightarrow t+0} \mathbf{V}_{\Phi}\left(f,\left(E_{t}^{+} \backslash\{t\}\right)_{\beta}^{+}\right)=\lim _{\beta \rightarrow t+0} \mathbf{V}_{\Phi}\left(f, E_{\beta}^{+}\right) \\
& =\mathbf{V}_{\Phi}(f, E)-\lim _{\beta \rightarrow t+0} \mathbf{V}_{\Phi}\left(f, E_{\beta}^{-}\right)=\mathbf{V}_{\Phi}\left(f, E_{t}^{+}\right)+\phi(t)-\phi(t+0) .
\end{aligned}
$$

(c) Прежде всего заметим, что

$$
\lim _{\substack{\alpha \rightarrow t-0 \\ \beta \rightarrow t+0}} \mathbf{V}_{\Phi}\left(f, E_{\alpha}^{\beta} \backslash\{t\}\right)=\inf _{\alpha, \beta} \mathbf{V}_{\Phi}\left(f, E_{\alpha}^{\beta} \backslash\{t\}\right),
$$


где инфимум берется по $\alpha \in E_{t}^{-}, \beta \in E_{t}^{+}, \alpha<t<\beta$. Покажем, что для любого $\varepsilon>0$ существуют $\alpha_{0}=\alpha_{0}(\varepsilon), \beta_{0}=\beta_{0}(\varepsilon) \in E, \alpha_{0}<t<\beta_{0}$, такие, что

$$
\mathbf{V}_{\Phi}\left(f, E_{\alpha}^{\beta} \backslash\{t\}\right) \leqslant U(\beta, \alpha)+\varepsilon \quad \forall \alpha \in E_{\alpha_{0}}^{t} \backslash\{t\}, \quad \forall \beta \in E_{t}^{\beta_{0}} \backslash\{t\} .
$$

По определению $\mathbf{V}_{\Phi}(f, E \backslash\{t\})$ для $\varepsilon>0$ найдем такое разбиение $T=\left\{t_{i}\right\}_{i=0}^{m}$ множества $E \backslash\{t\}$, что $t_{0}<t_{1}<\cdots<t_{k-1}<t<t_{k}<\cdots<t_{m-1}<t_{m}$ для некоторого $1 \leqslant k \leqslant m$ и $\mathbf{V}_{\Phi}(f, E \backslash\{t\}) \leqslant \sum_{i=1}^{m} U\left(t_{i}, t_{i-1}\right)+\varepsilon$. Положим $T_{1}=\left\{t_{i}\right\}_{i=0}^{k-1}, T_{2}=\left\{t_{i}\right\}_{i=k}^{m}, \alpha_{0}=t_{k-1}$ и $\beta_{0}=t_{k}$. Если теперь $\alpha, \beta \in E$ такие, что $\alpha_{0}<\alpha<t<\beta<\beta_{0}$, то, учитьвая, что $T_{1} \cup\{\alpha\} \in \mathscr{T}\left(E_{\alpha}^{-}\right)$и $T_{2} \cup\{\beta\} \in \mathscr{T}\left(E_{\beta}^{+}\right)$, в силу первого неравенства (12) получим

$$
\begin{aligned}
\mathbf{V}_{\Phi}(f, E \backslash\{t\}) & \leqslant V_{\Phi}\left[f ; T_{1}\right]+U\left(\alpha, t_{k-1}\right)+U(\beta, \alpha)+U\left(t_{k}, \beta\right)+V_{\Phi}\left[f ; T_{2}\right]+\varepsilon \\
& \leqslant \mathbf{V}_{\Phi}\left(f, E_{\alpha}^{-}\right)+U(\beta, \alpha)+\mathbf{V}_{\Phi}\left(f, E_{\beta}^{+}\right)+\varepsilon .
\end{aligned}
$$

Это доказьвает (13), если учесть, что лемма 2(c) дает равенство

$$
\mathbf{V}_{\Phi}(f, E \backslash\{t\})=\mathbf{V}_{\Phi}\left(f, E_{\alpha}^{-}\right)+\mathbf{V}_{\Phi}\left(f, E_{\alpha}^{\beta} \backslash\{t\}\right)+\mathbf{V}_{\Phi}\left(f, E_{\beta}^{+}\right)
$$

Остается перейти к пределу при $\alpha \rightarrow t-0, \beta \rightarrow t+0$ в $(13)$ и неравенстве $U(\beta, \alpha) \leqslant$ $\mathbf{V}_{\Phi}\left(f, E_{\alpha}^{\beta} \backslash\{t\}\right)$, справедливом для всех $\alpha \in E_{t}^{-}, \beta \in E_{t}^{+}, \alpha<t<\beta$.

(d) Из леммы 2(c) и равенства (14) для $\alpha, \beta \in E, \alpha<t<\beta$, имеем

$$
\mathbf{V}_{\Phi}(f, E)-\mathbf{V}_{\Phi}(f, E \backslash\{t\})=\phi(\beta)-\phi(\alpha)-\mathbf{V}_{\Phi}\left(f, E_{\alpha}^{\beta} \backslash\{t\}\right) .
$$

Равенство в (d) получается отсюда в пределе при $\alpha \rightarrow t-0, \beta \rightarrow t+0$.

СлЕДСТВИЕ 7. Отображсение $f \in B V_{\Phi}(E ; X)$ является

(а) непрерывным справа в точке $t \in E \backslash\{\sup E\}$ или слева в точке $t \in E \backslash\{\inf E\}$ тогда и только тогда, когда в точке $t$ этим же свойством обладает его функиия $\Phi$-вариации $\phi$;

(b) непрерывным на Е вне не более чем счетного множества точек.

СлЕДСТВие 8. Пусть $f \in B V_{\Phi}(E ; X), t \in E u[\Phi]=\lim _{\rho \rightarrow \infty} \Phi(\rho) / \rho<\infty$. Тогда

(a) если $t \in E_{t}^{-\prime}$, mo

$$
\mathbf{V}_{\Phi}\left(f, E_{t}^{-}\right)=\mathbf{V}_{\Phi}\left(f, E_{t}^{-} \backslash\{t\}\right)+[\Phi] \lim _{\alpha \rightarrow t-0} d(f(t), f(\alpha))
$$

(b) ecлut $t E_{t}^{+\prime}$, mo

$$
\mathbf{V}_{\Phi}\left(f, E_{t}^{+}\right)=\mathbf{V}_{\Phi}\left(f, E_{t}^{+} \backslash\{t\}\right)+[\Phi] \lim _{\beta \rightarrow t+0} d(f(\beta), f(t))
$$

(c) если жее $t \in E_{t}^{-\prime} \cap E_{t}^{+\prime}$, то дополнительно $\kappa(\mathrm{a}) u(\mathrm{~b})$ имеем

$$
\begin{aligned}
\mathbf{V}_{\Phi}(f, E)=\mathbf{V}_{\Phi}(f, E \backslash\{t\})+[\Phi]\left(\lim _{\alpha \rightarrow t-0} d(f(t), f(\alpha))\right. \\
\left.\quad+\lim _{\beta \rightarrow t+0} d(f(\beta), f(t))-\lim _{\substack{\alpha \rightarrow t-0 \\
\beta \rightarrow t+0}} d(f(\beta), f(\alpha))\right) .
\end{aligned}
$$


В случае полного метрического пространства $X$ в точках $t \in E_{t}^{ \pm \prime}$ существуют односторонние пределы $f(t \pm 0)=\lim _{\alpha \rightarrow t \pm 0} f(\alpha)$ соответственно, поэтому знаки пределов в следствии 8 можно “втащить" под знак метрики $d$.

Для $f \in G V_{\Phi}(E ; X)$ формула (а) из следствия 8 приобретает такой вид: если $t \in E_{t}^{-\prime}$, то

$$
p_{\Phi}\left(f, E_{t}^{-}\right) \leqslant p_{\Phi}\left(f, E_{t}^{-} \backslash\{t\}\right)+[\Phi] \lim _{\alpha \rightarrow t-0} d(f(t), f(\alpha))
$$

Доказательство этого проводится так же, как лемма 4(f). Аналогично модифицируются и другие формулы из следствия 8.

Следствие 8 в различной общности устанавливалось в работах [11], [12].

5. Обобщенный принцип выбора Хелли. Здесь мы распространим принцип выбора Хелли [28] на пространство $G V_{\Phi}(E ; X)$. Частные случаи приводимой ниже теоремы 9 устанавливались в работах [21], [11], [12], [14] и [17].

Теорема 9. Пусть $\Phi$ - выпуклая $\varphi$-функиия, $X$ - полное метрическое пространство, семейство $\mathscr{F} \subset X^{[a, b]}$ бесконечно и при любом $t \in[a, b]$ множество $\{f(t) \mid f \in \mathscr{F}\}$ предкомпактно в $X$. Eсли $\sup _{f \in \mathscr{F}} p_{\Phi}(f,[a, b])<\infty$, то $\mathscr{F}$ содержит последовательность, которая поточечно на $[a, b]$ сходится к некоторому отображению из $G V_{\Phi}([a, b] ; X)$. Если, в дополнение, $\Phi \in \mathcal{N}$, то эту последовательность в $\mathscr{F}$ можно выбрать сходящейся равномерно на $[a, b]$.

ДоказАТЕЛЬСтво. Для $f \in \mathscr{F}$ и $t \in[a, b]$ положим $\nu_{f}(t)=\mathbf{V}_{1}(f,[a, t])$. Семейство неубывающих функций $\left\{\nu_{f} \mid f \in \mathscr{F}\right\}$ бесконечно и ограничено в силу (11), а потому из принципа выбора Хелли для монотонных функций (см. [28], [21, гл. 8, §4]) следует, что найдутся последовательность $\left\{f_{n}\right\}_{n=1}^{\infty} \subset \mathscr{F}$ и неубывающая функция $\nu:[a, b] \rightarrow \mathbb{R}^{+}$ такие, что $\lim _{n \rightarrow \infty} \nu_{f_{n}}(t)=\nu(t)$ для всех $t \in[a, b]$. Поскольку множество точек разрьва функции $\nu$ не более чем счетно и при любом $t \in[a, b]$ множество $\left\{f_{n}(t)\right\}_{n=1}^{\infty}$ предкомпактно в $X$, не ограничивая общности, будем считать (извлекая, если необходимо, с помощью стандартного диагонального процесса подпоследовательность в $\left.\left\{f_{n}\right\}_{n=1}^{\infty}\right)$, что $f_{n}(s)$ сходится в $X$ к элементу $f(s)$ во всех рациональных точках $s \in[a, b]$, во всех точках $s$ разрьва функции $\nu$ и в точках $s=a$ и $s=b$.

Осталось показать, что $f_{n}(t)$ имеет предел в $X$ в любой иррациональной точке $a<$ $t<b$ непрерьвности функции $\nu$. Для $\varepsilon>0$ найдем такое рациональное число $a<s<t$, что $0 \leqslant \nu(t)-\nu(s) \leqslant \varepsilon / 9$, и выберем номер $N_{0} \in \mathbb{N}$ так, чтобы $\left|\nu_{f_{n}}(t)-\nu(t)\right| \leqslant \varepsilon / 9$ и $\left|\nu_{f_{n}}(s)-\nu(s)\right| \leqslant \varepsilon / 9$ при $n \geqslant N_{0}$. Тогда

$$
d\left(f_{n}(t), f_{n}(s)\right) \leqslant\left|\nu_{f_{n}}(t)-\nu(t)\right|+(\nu(t)-\nu(s))+\left|\nu(s)-\nu_{f_{n}}(s)\right| \leqslant \frac{\varepsilon}{3}, \quad n \geqslant N_{0} .
$$

Если $N_{1} \in \mathbb{N}$ такой номер, что $d\left(f_{n}(s), f_{m}(s)\right) \leqslant \varepsilon / 3$ при $n, m \geqslant N_{1}$, то для всех $n, m \geqslant \max \left\{N_{0}, N_{1}\right\}$ будем иметь

$$
d\left(f_{n}(t), f_{m}(t)\right) \leqslant d\left(f_{n}(t), f_{n}(s)\right)+d\left(f_{n}(s), f_{m}(s)\right)+d\left(f_{m}(s), f_{m}(t)\right) \leqslant \varepsilon .
$$

Таким образом, последовательность $\left\{f_{n}(t)\right\}_{n=1}^{\infty}$ фундаментальна в $X$. Остается положить $f(t)=\lim _{n \rightarrow \infty} f_{n}(t)$ и применить лемму $4(\mathrm{e})$.

Если известно, что $\Phi \in \mathscr{N}$, то отрезок $[a, b]$ можно заменить на компакт $E$ в $\mathbb{R}$. В этом случае равномерная сходимость некоторой последовательности из $\mathscr{F}$ вытекает из 
теоремы Арцела-Асколи, поскольку в силу леммы 4(а) и равенства (2) семейство $\mathscr{F}$ равностепенно непрерьвно.

Покажем, что условия теоремы 9 не могут быть ослаблены. Для вьпуклой $\varphi$-функции $\varphi$ обозначим через $\ell_{\varphi}$ пространство всех последовательностей $x=\left\{x_{n}\right\}_{n=1}^{\infty} \in \mathbb{R}^{\mathbb{N}}$ таких, что существует число $\lambda>0$ (зависящее от $x$ ), для которого $\sum_{n=1}^{\infty} \varphi\left(\lambda\left|x_{n}\right|\right)<\infty$ (см. $[19, \S \S 3,10])$. Пространство $\ell_{\varphi}$ является бесконечномерной банаховой алгеброй относительно нормы $\|x\|_{\varphi}=\inf \left\{\lambda>0 \mid \sum_{n=1}^{\infty} \varphi\left(\left|x_{n}\right| / \lambda\right) \leqslant 1\right\}$ и имеет стандартный базис $\left\{e_{n}\right\}_{n=1}^{\infty}$, где $e_{n}=\left\{x_{i}\right\}_{i=1}^{\infty}$ с $x_{i}=0$ при $i \neq n$ и $x_{n}=1$. Для выпуклой $\varphi$-функции $\Phi, f \in B V_{\Phi}([0,1] ; \mathbb{R}), f \neq 0$, и $n \in \mathbb{N}$ положим $f_{n}(t)=f(t) e_{n}, t \in[0,1]$, так что $f_{n}:[0,1] \rightarrow \ell_{\varphi}$ и $\mathbf{V}_{\Phi}\left(f_{n},[0,1]\right)=\mathbf{V}_{\Phi}(f,[0,1]) / \varphi^{-1}(1)$. Таким образом, последовательность $\left\{f_{n}\right\}_{n=1}^{\infty}$ имеет равномерно ограниченную $\Phi$-вариацию, но множества $\left\{f_{n}(t)\right\}_{n=1}^{\infty}$ не предкомпактны в $\ell_{\varphi}$, поэтому обобщенньй принцип выбора Хелли нарушается: никакая подпоследовательность последовательности $\left\{f_{n}\right\}_{n=1}^{\infty}$ не сходится в $\ell_{\varphi}$ ни в одной точке $t \in[0,1]$.

При помощи стандартного диагонального метода принцип выбора Хелли распространяется на семейства отображений, областью определения которых служат ограниченные или неограниченные интервалы или полуинтервалы.

6. Селекции многозначных отображений обобщенной Ф-вариации. Многозначное отображение $F: E \rightarrow \mathrm{c}(X)$ называется липиииевы. о, отображсением конечной $\Phi$-вариации или отображсением конечной обобщенной $\Phi$-вариации, если оно обладает этим свойством (в смысле п. 2) относительно хаусдорфовой метрики $D$, порожденной метрикой $d$ на $X$. Соответственно пишем

$$
F \in \operatorname{Lip}(E ; \mathrm{c}(X)), \quad F \in B V_{\Phi}(E ; \mathrm{c}(X)), \quad F \in G V_{\Phi}(E ; \mathrm{c}(X)) .
$$

Заметим, что на пространстве $\mathrm{c}(X)$ хаусдорфова топология (т.е. топология, порожденная хаусдорфовой метрикой) зависит только от топологии $X$ (а не от метрики на $X$, см., например, [4, следствие II-7]).

Напомним, что без условия вьпуклости образов многозначного отображения $F: I=$ $[a, b] \rightarrow \mathrm{c}\left(\mathbb{R}^{n}\right), n=1,2$, непрерывных селекций может не иметь непрерьвное отображение $F$ (см. [1], [6], [29]) и даже отображение $F$ с компактньми образами в $\mathbb{R}^{2}$, удовлетворяющее при любом $0<\alpha<1$ условию Гёльдера $D(F(t), F(s)) \leqslant C|t-s|^{\alpha}$, $t, s \in I$ (см. [30]). Отметим также, что для многозначных отображений $F$, определенных на промежутке в $\mathbb{R}$ и принимающих в топологическом пространстве связные значения, образующие равностепенно локально связное семейство множеств, непрерьвные селекции существуют без каких-либо метрических ограничений - достаточно, чтобы $F$ было полунепрерьвно снизу (см. [5]).

Теорема 10. Пусть $(X, d)$ - метрическое пространство, $\Phi$ - выпуклая $\varphi$-функиия, $F \in G V_{\Phi}([a, b] ; \mathrm{c}(X))$ - компактнозначное многозначное отображсение конечной обобщенной $\Phi$-вариачии, $t_{0} \in[a, b]$ и $x_{0} \in X$. Тогда $F$ обладает селекиией $f \in$ $G V_{\Phi}([a, b] ; X)$ такой, что

$$
\begin{gathered}
d\left(x_{0}, f\left(t_{0}\right)\right)=\operatorname{dist}\left(x_{0}, F\left(t_{0}\right)\right), \quad p_{\Phi}(f,[a, b]) \leqslant p_{\Phi}(F,[a, b]) \\
\mathbf{V}_{1}(f,[a, b]) \leqslant \mathbf{V}_{1}(F,[a, b]) .
\end{gathered}
$$


Если, кроме того, $F$ непрерывно, то и эту селекиию $f$ также можсно выбрать непрерывной, а если $F \in \operatorname{Lip}([a, b] ; \mathrm{c}(X))$, то и селекиию $f \in \operatorname{Lip}([a, b] ; X)$ можно вьбрать такой, что $\mathbf{L}(f) \leqslant \mathbf{L}(F)$. Отметим, в частности, что если $x_{0} \in F\left(t_{0}\right)$, mo $f\left(t_{0}\right)=x_{0}$.

Прежде чем доказывать эту теорему, установим следующую лемму.

Лемма 11. Если $F \in B V_{1}(E ; \mathrm{c}(X))$, әде $\varnothing \neq E \subset \mathbb{R} u(X, d)$ - метрическое пространство, то образ $\operatorname{Im}(F)=F(E)$ отображсения $F$, определяемый правилом $\operatorname{Im}(F)=\bigcup_{t \in E} F(t)$, является вполне ограниченным и сепарабельным подмножеством $X$; а если, к тому же, $X$ полное, то $\operatorname{Im}(F)$ предкомпактно.

ДокАЗАТЕЛЬСтво. Для $\varepsilon>0, x \in X$ и компактного множества $K \subset X$ положим $B_{\varepsilon}(x)=\{y \in X \mid d(y, x)<\varepsilon\}$ и $\mathscr{O}_{\varepsilon}(K)=\{y \in X \mid \operatorname{dist}(y, K)<\varepsilon\}$. Чтобы доказать, что $\operatorname{Im}(F)$ вполне ограничено, нужно показать, что для любого $\varepsilon>0$ множество $\operatorname{Im}(F)$ можно покрыть конечным числом шаров $B_{\varepsilon}(\cdot)$ с центрами в $\operatorname{Im}(F)$, т.е. что найдутся номер $N$, зависящий от $\varepsilon$, и точки $t_{n} \in E$ и $x_{n} \in F\left(t_{n}\right), n=1, \ldots, N$, такие, что $\operatorname{Im}(F) \subset \bigcup_{n=1}^{N} B_{\varepsilon}\left(x_{n}\right)$. Учитьвая, что для компактного $K \subset X$ имеет место равенство $\mathscr{O}_{\varepsilon}(K)=\bigcup_{x \in K} B_{\varepsilon}(x)$, найдем, что условие вполне ограниченности $\operatorname{Im}(F)$ эквивалентно тому, что для любого $\varepsilon>0$ существуют номер $N$ и точки $t_{n} \in E, n=1, \ldots, N$, такие, что $\operatorname{Im}(F) \subset \bigcup_{n=1}^{N} \mathscr{O}_{\varepsilon}\left(F\left(t_{n}\right)\right)$. От противного предположим, что $\varepsilon>0$ - такое число, что утверждение в последнем предложении нарушается. Построим последовательность $\left\{x_{n}\right\}_{n=0}^{\infty} \subset \operatorname{Im}(F)$ по индукции следующим образом. Произвольно зафиксируем $t_{0} \in E$ и $x_{0} \in F\left(t_{0}\right)$, выберем $x_{1} \in \operatorname{Im}(F)$ так, чтобы $x_{1} \notin \mathscr{O}_{\varepsilon}\left(F\left(t_{0}\right)\right)$, и будем считать, что $x_{1} \in F\left(t_{1}\right)$ для некоторого $t_{1} \in E$. Если точки $x_{0}, x_{1}, \ldots, x_{n-1} \in \operatorname{Im}(F)$, $n \geqslant 2$, уже определены, причем $x_{j} \in F\left(t_{j}\right)$ для $t_{j} \in E, j=0,1, \ldots, n-1$, то выберем точку $x_{n} \in \operatorname{Im}(F) \backslash \bigcup_{j=0}^{n-1} \mathscr{O}_{\varepsilon}\left(F\left(t_{j}\right)\right)$ и будем считать, что $x_{n} \in F\left(t_{n}\right)$ для некоторого $t_{n} \in E$. Поскольку для $n>k D\left(F\left(t_{n}\right), F\left(t_{k}\right)\right) \geqslant \operatorname{dist}\left(x_{n}, F\left(t_{k}\right)\right) \geqslant \varepsilon$, то $t_{n} \neq t_{k}$, поэтому без ограничения общности можно считать, что $t_{n-1}<t_{n}$ для всех $n \in \mathbb{N}$. Тогда для разбиения $T_{m}=\left\{t_{i}\right\}_{i=0}^{m}$ множества $E$ имеем

$$
\mathbf{V}_{1}(F, E) \geqslant \sum_{i=1}^{m} D\left(F\left(t_{i}\right), F\left(t_{i-1}\right)\right) \geqslant m \varepsilon
$$

а в силу произвольности $m$ заключаем, что $\mathbf{V}_{1}(F, E)=\infty$, что противоречит предположению. Известно, что в метрическом пространстве вполне ограниченное множество сепарабельно, а в полном метрическом пространстве оно предкомпактно.

ДокАЗАТЕЛЬСТво ТЕОРЕмЫ 10. Установим эту теорему вначале для $F$ из класса $B V_{\Phi}([a, b] ; \mathrm{c}(X))$. Для $n \in \mathbb{N}$ рассмотрим разбиение $T_{n}=\left\{t_{i}^{n}\right\}_{i=0}^{n} \in \mathscr{T}_{a}^{b}$ со следующими двумя свойствами:

1) $t_{0} \in T_{n}$, т.е. $t_{0}=t_{k(n)}^{n}$ для некоторого $k(n) \in\{0,1, \ldots, n\}$;

2) если $I_{i}^{n}=\left[t_{i-1}^{n}, t_{i}^{n}\right]$ и $\left|I_{i}^{n}\right|=t_{i}^{n}-t_{i-1}^{n}, i=1, \ldots, n$, то $\lim _{n \rightarrow \infty} \max _{1 \leqslant i \leqslant n}\left|I_{i}^{n}\right|=0$. В силу компактности множества $F\left(t_{0}\right)$ выберем элемент $y_{0} \in F\left(t_{0}\right)$ так, чтобы $d\left(x_{0}, y_{0}\right)=$ $\operatorname{dist}\left(x_{0}, F\left(t_{0}\right)\right)$. По индукции определим элементы $x_{i}^{n} \in F\left(t_{i}^{n}\right), i=0,1, \ldots, n$, следующим образом. Пусть вначале $a<t_{0}<b$.

а) Положим $x_{k(n)}^{n}=y_{0}$. 
b) Если $i \in\{1, \ldots, k(n)\}$ и элемент $x_{i}^{n} \in F\left(t_{i}^{n}\right)$ уже определен, выберем элемент $x_{i-1}^{n} \in F\left(t_{i-1}^{n}\right)$ так, чтобы $d\left(x_{i}^{n}, x_{i-1}^{n}\right)=\operatorname{dist}\left(x_{i}^{n}, F\left(t_{i-1}^{n}\right)\right)$.

c) Если $i \in\{k(n)+1, \ldots, n\}$ и элемент $x_{i}^{n} \in F\left(t_{i}^{n}\right)$ уже определен, выберем элемент $x_{i}^{n} \in F\left(t_{i}^{n}\right)$ так, чтобы $d\left(x_{i-1}^{n}, x_{i}^{n}\right)=\operatorname{dist}\left(x_{i-1}^{n}, F\left(t_{i}^{n}\right)\right)$.

Для $t_{0}=a$, т.е. $k(n)=0$, определяем $x_{i}^{n}$ согласно а) и с), а если $t_{0}=b$, так что $k(n)=n$, следуем a) и b). Благодаря b), с) и (1) имеем

$$
d\left(x_{i}^{n}, x_{i-1}^{n}\right) \leqslant D\left(F\left(t_{i}^{n}\right), F\left(t_{i-1}^{n}\right)\right), \quad n \in \mathbb{N}, \quad i=1, \ldots, n .
$$

В силу $(9) \mathbf{V}_{1}(F,[a, b])<\infty$, поэтому по лемме 11 образ $\operatorname{Im}(F)$ отображения $F$ (вполне) ограничен в $X$. Обозначим через $Y=C_{b}(\operatorname{Im}(F) ; \mathbb{R})$ банахово пространство всех непрерьвных ограниченных функций из $\operatorname{Im}(F)$ в $\mathbb{R}$ с равномерной нормой

$$
\|y\|=\sup _{x \in \operatorname{Im}(F)}|y(x)|, \quad y \in Y .
$$

Отображение $\operatorname{Im}(F) \ni x \mapsto R(x) \in Y$, где $R(x)\left(x^{\prime}\right)=d\left(x, x^{\prime}\right), x^{\prime} \in \operatorname{Im}(F)$, определяет (согласно Куратовскому) изометрическое вложение множества $\operatorname{Im}(F)$ в пространство $Y$, так что, в частности, $\left\|R(x)-R\left(x^{\prime}\right)\right\|=d\left(x, x^{\prime}\right)$ для всех $x, x^{\prime} \in \operatorname{Im}(F)$.

Для $n \in \mathbb{N}$ определим отображение $f_{n}:[a, b] \rightarrow Y$ правилом

$$
f_{n}(t)=R\left(x_{i-1}^{n}\right)+\frac{\left(t-t_{i-1}^{n}\right)\left(R\left(x_{i}^{n}\right)-R\left(x_{i-1}^{n}\right)\right)}{\left|I_{i}^{n}\right|}, \quad t \in I_{i}^{n}, \quad i=1, \ldots, n .
$$

Отметим, что $f_{n}\left(t_{0}\right)=R\left(y_{0}\right), f_{n}\left(t_{i}^{n}\right)=R\left(x_{i}^{n}\right), n \in \mathbb{N}, i=0,1, \ldots, n$, поэтому из леммы $2(\mathrm{c}),(15),(16)$ и изометричности $R$ для всех $n \in \mathbb{N}$ находим, что

$$
\begin{aligned}
\mathbf{V}_{\Phi}\left(f_{n},[a, b]\right) & =\sum_{i=1}^{n} \mathbf{V}_{\Phi}\left(f_{n}, I_{i}^{n}\right)=\sum_{i=1}^{n} \Phi\left(\frac{d\left(x_{i}^{n}, x_{i-1}^{n}\right)}{\left|I_{i}^{n}\right|}\right)\left|I_{i}^{n}\right| \\
& \leqslant \sum_{i=1}^{n} \Phi\left(\frac{\left(D\left(F\left(t_{i}^{n}\right), F\left(t_{i-1}^{n}\right)\right)\right.}{\left|I_{i}^{n}\right|}\right)\left|I_{i}^{n}\right| \leqslant \mathbf{V}_{\Phi}(F,[a, b]) .
\end{aligned}
$$

В силу леммы $4(\mathrm{c})$ получаем, что $p_{\Phi}\left(f_{n},[a, b]\right) \leqslant \max \left\{1, \mathbf{V}_{\Phi}(F,[a, b])\right\}, n \in \mathbb{N}$, а из неравенства $(17)$ при $\Phi(\rho)=\rho$ следует также, что $\mathbf{V}_{1}\left(f_{n},[a, b]\right) \leqslant \mathbf{V}_{1}(F,[a, b])$.

Для $t \in[a, b]$ покажем, что последовательность $\left\{f_{n}(t)\right\}_{n=1}^{\infty}$ предкомпактна в $Y$. Пусть вначале $\Phi \in \mathcal{N}$, так что $F$ (абсолютно) непрерьвно на $[a, b]$ относительно $D$. Для любого $n \in \mathbb{N}$ существует номер $i(n) \in\{1, \ldots, n\}$ (зависящий также от $t$ ) такой, что $t \in I_{i(n)}^{n}$, поэтому из условия 2) следует, что $t_{i(n)}^{n} \rightarrow t$ и $t_{i(n)-1}^{n} \rightarrow t$ при $n \rightarrow \infty$. Выберем элемент $x_{n}(t) \in F(t)$ так, чтобы $d\left(x_{i(n)-1}^{n}, x_{n}(t)\right)=\operatorname{dist}\left(x_{i(n)-1}^{n}, F(t)\right), n \in \mathbb{N}$. Поскольку $F(t)$ компактно, можно считать (переходя к подпоследовательности), что $x_{n}(t)$ сходится в $X$ к некоторому элементу $x(t) \in F(t)$. Тогда в силу (16) и (15)

$$
\begin{aligned}
\left\|f_{n}(t)-R(x(t))\right\| \leqslant & \left\|f_{n}(t)-R\left(x_{n}(t)\right)\right\|+\left\|R\left(x_{n}(t)\right)-R(x(t))\right\| \\
\leqslant & \left\|R\left(x_{i(n)-1}^{n}\right)-R\left(x_{n}(t)\right)\right\|+\left\|R\left(x_{i(n)}^{n}\right)-R\left(x_{i(n)-1}^{n}\right)\right\| \\
& \quad+\left\|R\left(x_{n}(t)\right)-R(x(t))\right\| \\
\leqslant & D\left(F\left(t_{i(n)-1}^{n}\right), F(t)\right)+D\left(F\left(t_{i(n)}^{n}\right), F\left(t_{i(n)-1}^{n}\right)\right)+d\left(x_{n}(t), x(t)\right) \\
\rightarrow & 0, \quad n \rightarrow \infty
\end{aligned}
$$


а это означает, что

$$
f_{n}(t) \rightarrow R(x(t)) \quad \text { в } Y \text { при } n \rightarrow \infty,
$$

и доказывает предкомпактность $\left\{f_{n}(t)\right\}_{n=1}^{\infty}$. В случае, когда $[\Phi]<\infty$, перенумеруем множество точек разрьва $F$, которое в силу следствия $7(\mathrm{~b})$ не более чем счетно. В каждое рассмотренное выше разбиение $T_{n}$ добавим $n$ первых точек разрыва $F$ и получившееся разбиение по-прежнему обозначим через $T_{n}$, так что новое разбиение $T_{n}$ снова удовлетворяет условиям 1) и 2). Предкомпактность $\left\{f_{n}(t)\right\}_{n=1}^{\infty}$ в точке $t$ непрерьвности $F$ вытекает из приведенных выше рассуждений. Если же $t \in[a, b]$ есть точка разрыва $F$, то найдется номер $N(t) \in \mathbb{N}$ такой, что $t \in T_{n}$ для для всех $n \geqslant N(t)$, т.е. $t=t_{\ell(n)}^{n}$ для некоторого номера $\ell(n) \in\{0,1, \ldots, n\}$, также зависящего от $t$. По построению $x_{\ell(n)}^{n} \in F\left(t_{\ell(n)}^{n}\right)=F(t), n \geqslant N(t)$, поэтому в силу компактности множества $F(t)$ некоторая подпоследовательность в $\left\{x_{\ell(n)}^{n}\right\}_{n=1}^{\infty}$ (для которой сохраним это же обозначение) сходится в $X$ к некоторой точке $x(t) \in F(t)$. Поскольку $f_{n}(t)=R\left(x_{\ell(n)}^{n}\right)$ при $n \geqslant N(t)$, то снова вьполнено условие (18).

Применяя обобщенный принцип выбора Хелли (теорема 9), найдем подпоследовательность в $\mathscr{F}=\left\{f_{n}\right\}_{n=1}^{\infty}$, поточечно на $[a, b]$ сходящуюся в пространстве $Y$ к некоторому отображению $g:[a, b] \rightarrow Y$ вида (см. (18)) $g(t)=R(f(t)), t \in[a, b]$, где $f(t) \in F(t)$ для всех $t \in[a, b]$, причем $g\left(t_{0}\right)=R\left(y_{0}\right)$, а лемма $2(\mathrm{~d})$ и неравенство (17) гарантируют, что $\mathbf{V}_{\Phi}(g,[a, b]) \leqslant \mathbf{V}_{\Phi}(F,[a, b])$ и $\mathbf{V}_{1}(g,[a, b]) \leqslant \mathbf{V}_{1}(F,[a, b])$. Поскольку $R$ - изометрия, заключаем, что $f\left(t_{0}\right)=y_{0}, \mathbf{V}_{\Phi}(f,[a, b]) \leqslant \mathbf{V}_{\Phi}(F,[a, b])$ и $\mathbf{V}_{1}(f,[a, b]) \leqslant \mathbf{V}_{1}(F,[a, b])$.

Если известно, что $F \in \operatorname{Lip}([a, b] ; \mathrm{c}(X))$, то в силу (16) и (15) для $n \in \mathbb{N}$ и $i=1, \ldots, n$ достаточно дополнительно учесть неравенства

$$
\left\|f_{n}(t)-f_{n}(s)\right\| \leqslant \frac{|t-s| d\left(x_{i}^{n}, x_{i-1}^{n}\right)}{\left|I_{i}^{n}\right|} \leqslant \mathbf{L}(F)|t-s|, \quad t, s \in I_{i}^{n} .
$$

Тогда селекция $f \in \operatorname{Lip}([a, b] ; X)$ отображения $F$ такова, что $\mathbf{L}(f) \leqslant \mathbf{L}(F)$.

Пусть $F \in B V_{\Phi}([a, b] ; \mathrm{c}(X))$ непрерывно. По лемме $5($ a) имеем разложение $F=G \circ \nu$, где функция $\nu(t)=\mathbf{V}_{1}(F,[a, t]), t \in[a, b]$, принадлежит пространству $B V_{\Phi}([a, b] ; \mathbb{R})$ и непрерьвна, а многозначное отображение $G$ из $\operatorname{Lip}([0, \ell] ; \mathrm{c}(X))$ обладает следующими свойствами: $\ell=\mathbf{V}_{1}(F,[a, b])=\mathbf{V}_{1}(\nu,[a, b]), \mathbf{L}(G) \leqslant 1$ и $\mathbf{V}_{\Phi}(\nu,[a, b])=\mathbf{V}_{\Phi}(F,[a, b])$. Положим $\tau_{0}=\nu\left(t_{0}\right) \in[0, \ell]$, так что $G\left(\tau_{0}\right)=F\left(t_{0}\right)$. Существует $g \in \operatorname{Lip}([0, \ell] ; X)$ такое, что $d\left(x_{0}, g\left(\tau_{0}\right)\right)=\operatorname{dist}\left(x_{0}, G\left(\tau_{0}\right)\right), g(\tau) \in G(\tau)$ для всех $\tau \in[0, \ell]$ и $\mathbf{L}(g) \leqslant \mathbf{L}(G) \leqslant 1$. Полагая $f=g \circ \nu$, найдем, что $f \in B V_{\Phi}([a, b] ; X)$ непрерывно, $f\left(t_{0}\right)=g\left(\tau_{0}\right), f(t) \in F(t)$ для всех точек $t \in[a, b]$ и имеют место следующие неравенства: $\mathbf{V}_{1}(f,[a, b]) \leqslant \mathbf{V}_{1}(F$, $[a, b])$ и

$$
\mathbf{V}_{\Phi}(f,[a, b])=\mathbf{V}_{\Phi}(g \circ \nu,[a, b]) \leqslant \mathbf{V}_{\Phi}(\nu,[a, b])=\mathbf{V}_{\Phi}(F,[a, b]) .
$$

Пусть, наконец, $F \in G V_{\Phi}([a, b] ; X)$. Полагая $\lambda=p_{\Phi}(F,[a, b])$, в силу леммы 4(a) можно считать, что $\lambda>0$, так что $\mathbf{V}_{\Phi_{\lambda}}(F,[a, b]) \leqslant 1$ по лемме $4(\mathrm{~b})$. По доказанному выше существует селекция $f \in B V_{\Phi_{\lambda}}([a, b] ; X)$ такая, что $\mathbf{V}_{\Phi_{\lambda}}(f,[a, b]) \leqslant \mathbf{V}_{\Phi_{\lambda}}(F,[a, b])$. Следовательно, $p_{\Phi}(f,[a, b]) \leqslant \lambda$.

Рассмотрим банахово пространство $\ell_{1}$ суммируемых последовательностей с нормой $\|x\|_{1}=\sum_{n=1}^{\infty}\left|x_{n}\right|, x=\left\{x_{n}\right\}_{n=1}^{\infty} \in \ell_{1}$. Для $t \in[0,1]$ определим многозначное отображение

$$
F(t)=\left\{x \in \ell_{1}:\|x\|_{1}=1 \text { и } x_{1}=t\right\} .
$$


Тогда хаусдорфова метрика $D(F(t), F(s))=2|t-s|$ при $t, s \in[0,1]$, и любое отображение вида

$$
f(t)=\left(t,(1-t)\left\{x_{n}\right\}_{n=2}^{\infty}\right), \quad t \in[0,1], \quad \text { где } \sum_{n=2}^{\infty}\left|x_{n}\right|=1,
$$

является липшицевой селекцией $F$. Если теперь $\nu \in G V_{\Phi}([a, b] ; \mathbb{R})$ и $\nu([a, b])=[0,1]$, то $f \circ \nu$ есть селекция $F \circ \nu$ в классе отображений $G V_{\Phi}([a, b] ; \cdot)$.

СлЕДСТВИЕ 12. Теорема 10 справедлива, если в ней заменить $[a, b]$ на $\mathbb{R}$.

ДокАЗАтЕльство. Для начала пусть многозначное отображение $F$ принадлежит $B V_{\Phi}(\mathbb{R} ; \mathrm{c}(X))$. Пусть $\left\{t_{k}\right\}_{k \in \mathbb{Z}} \subset \mathbb{R}$ - возрастающая последовательность такая, что $t_{k} \rightarrow \infty$ и $t_{-k} \rightarrow-\infty$ при $k \rightarrow \infty$. Полагая $I_{k}=\left[t_{k}, t_{k+1}\right]$ для $k \in \mathbb{Z}$, применим теорему 10 на отрезке $I_{0}$ и найдем селекцию $f_{0} \in B V_{\Phi}\left(I_{0} ; X\right)$ отображения $F$ (точнее, сужения $\left.F\right|_{I_{0}}$ отображения $F$ на отрезок $\left.I_{0}\right)$ такую, что $d\left(x_{0}, f_{0}\left(t_{0}\right)\right)=\operatorname{dist}\left(x_{0}, F\left(t_{0}\right)\right)$, $\mathbf{V}_{\Phi}\left(f_{0}, I_{0}\right) \leqslant \mathbf{V}_{\Phi}\left(F, I_{0}\right)$ и $\mathbf{V}_{1}\left(f_{0}, I_{0}\right) \leqslant \mathbf{V}_{1}\left(F, I_{0}\right)$. “Двигаясь по отрезкам $I_{k}$ вправо" от точки $t_{1}$, будем последовательно применять теорему 10: на отрезке $I_{1}$ с начальным условием $x_{0}=f_{0}\left(t_{1}\right) \in F\left(t_{1}\right), \ldots$, на отрезке $I_{k}$ с начальным условием $x_{0}=f_{k-1}\left(t_{k}\right) \in$ $F\left(t_{k}\right), k \in \mathbb{N}$. В результате для любого $k \in \mathbb{N}$ найдем селекцию $f_{k} \in B V_{\Phi}\left(I_{k} ; X\right)$ отображения $\left.F\right|_{I_{k}}$ такую, что

$$
f_{k}\left(t_{k}\right)=f_{k-1}\left(t_{k}\right), \quad \mathbf{V}_{\Phi}\left(f_{k}, I_{k}\right) \leqslant \mathbf{V}_{\Phi}\left(F, I_{k}\right), \quad \mathbf{V}_{1}\left(f_{k}, I_{k}\right) \leqslant \mathbf{V}_{1}\left(F, I_{k}\right) .
$$

Аналогично будем “двигаться по отрезкам $I_{k}$ влево” от точки $t_{0}$. Тогда при любом $k \in \mathbb{Z}$ на отрезке $I_{k}$ имеется селекция $f_{k}$ отображения $F$ такая, что справедливы соотношения (19). Если $t \in \mathbb{R}$, так что $t \in I_{k}$ при некотором $k \in \mathbb{Z}$, положим $f(t)=f_{k}(t)$. Ясно, что отображение $f: \mathbb{R} \rightarrow X$ является селекцией $F$ на $\mathbb{R}, f\left(t_{0}\right)=f_{0}\left(t_{0}\right)$, а в силу леммы $2(\mathrm{~h}, \mathrm{c})$ имеем

$$
\begin{aligned}
\mathbf{V}_{\Phi}(f, \mathbb{R}) & =\lim _{k \rightarrow \infty} \mathbf{V}_{\Phi}\left(f,\left[t_{-k}, t_{k}\right]\right)=\lim _{k \rightarrow \infty} \sum_{i=-k}^{k-1} \mathbf{V}_{\Phi}\left(f_{i}, I_{i}\right) \\
& \leqslant \lim _{k \rightarrow \infty} \sum_{i=-k}^{k-1} \mathbf{V}_{\Phi}\left(F, I_{i}\right)=\lim _{k \rightarrow \infty} \mathbf{V}_{\Phi}\left(F,\left[t_{-k}, t_{k}\right]\right)=\mathbf{V}_{\Phi}(F, \mathbb{R}),
\end{aligned}
$$

и, аналогично, $\mathbf{V}_{1}(f, \mathbb{R}) \leqslant \mathbf{V}_{1}(F, \mathbb{R})$.

Если теперь $F \in G V_{\Phi}(\mathbb{R} ; X)$, то для $\lambda=p_{\Phi}(f, \mathbb{R})>0$ имеем $\mathbf{V}_{\Phi_{\lambda}}(F, \mathbb{R}) \leqslant 1$, и остается воспользоваться только что доказанным результатом.

Утверждение, подобное следствию 12 , справедливо и для ограниченных или неограниченных интервалов и полуинтервалов вещественной прямой.

Отметим, что липшицевы селекции для липшицевых многозначных отображений в различных контекстах устанавливались в работах [7], [8], [10]-[12], [16], [17], [31].

Отображение $f:[a, b] \rightarrow X$ называется правильным, если оно имеет не более чем счетное множество точек разрыва, в которых сушествуют односторонние пределы слева и справа. Говорят, что последовательность $f_{n}:[a, b] \rightarrow X, n \in \mathbb{N}$, является представлением Кастэна для многозначного отображения $F:[a, b] \rightarrow \mathrm{c}(X)$, если для почти всех $t \in[a, b]$ последовательность $\left\{f_{n}(t)\right\}_{n=1}^{\infty}$ плотна в $F(t)$. Напомним [4, теорема III.8], что измеримость отображения $F$ с замкнутыми образами эквивалентна наличию для него представления Кастэна из последовательности измеримых селекций. Приводимое ниже следствие базируется на теореме 10 и устанавливается подобно теореме Д1.9 из [10]. 
СлЕДСТВИЕ 13. Пусть $X$ - полное метрическое пространство, $\Phi$ - выпуклая ч-функиия $и F \in G V_{\Phi}([a, b] ; \mathrm{c}(X))$. Тогда

(a) для любой измеримой селекиии $f$ отображсния $F$ найдется последовательность правильных селекиий $F$, которая почти всюдуна $[a, b]$ сходится $\kappa f$;

(b) существует последовательность правильных селекиий $F$, которая является представлением Кастәна для отображения $F$.

7. Селекции многозначных отображений на произведении двух пространств. Пусть $X$ и $Y$ - два (хаусдорфовых) топологических пространства. Напомним, что многозначное отображение $\Gamma$ из $X$ в $Y$ называется:

полунепрерывным снизу (сокращенно п.н.сн.) на $X$, если для любого открытого множества $\mathscr{U} \subset Y$ его предобраз $\Gamma^{-}(\mathscr{U})=\{x \in X \mid \Gamma(x) \cap \mathscr{U} \neq \varnothing\}$ открыт в $X$;

полунепрерывным сверху (n.н.св.) на $X$, если $\Gamma^{-}(\mathscr{U})$ замкнут в $X$ для любого замкнутого $\mathscr{U} \subset Y$;

непрерывны.м на $X$, если Г является п.н.сн. и п.н.св. на $X$ одновременно.

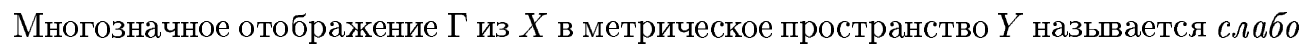
n.н.cв., если для любого замкнутого шара $\mathscr{U}$ в $Y$ предобраз $\Gamma^{-}(\mathcal{U})$ замкнут в $X$. Если метрическое пространство $Y$ обладает свойством:

замыкание в $Y$ любого открытого шара есть замкнутый шар,

то из слабой п.н.св. многозначного отображения Г вытекает его п.н.св. Например, свойством (20) обладает любое линейное метрическое пространство.

Напомним также, что топологическое пространство называется әкстремально несвязным (см. [32,§ 46.VI]), если замыкание всякого открытого в нем множества открыто.

Нам потребуется следующий результат из [33] (другое болеепростое доказательство этого результата содержится в [34]).

ЛЕмма 14. Пусть $X$ - әкстремально несвязное хаусдорфово топологическое пространство, $Y$ - регулярное хаусдорфово $\mathscr{T}_{3}$-пространство и Г- п.н.св. многозначное отображсение из $X$ в $Y$ с компактными значениями. Тогда Г обладает непрерывной селекиией $\gamma: X \rightarrow Y$.

Пусть $I$ - промежуток (связное подмножество $\mathbb{R}$ ) и многозначное отображение $H=$ $H(t, x)$ действует из $I \times X$ в $Y$. Для фиксированного $x \in X$ определим многозначное отображение $H(\cdot, x)$ из $I$ в $Y$ правилом $H(\cdot, x)(t)=H(t, x), t \in I$, а если $t \in I$ фиксировано, аналогично полагаем $H(t, \cdot)(x)=H(t, x), x \in X$, так что $H(t, \cdot)$ отображает $X$ в подмножества $Y$.

Основной результат этого пункта - следующая

ТЕОремА 15. Пусть $I \subset \mathbb{R}$ - промежуток, $X$ - әкстремально несвязное хаусдорфово топологическое пространство, $(Y, d)$ - полное метрическое пространство, удовлетворяющее условию (20), $D$ - хаусдорфова метрика на с $(Y)$, порохденная $d, t_{0} \in I, \eta: X \rightarrow Y-$ непрерывное отображсение, $\Phi \in \mathcal{N} u H: I \times X \rightarrow \mathrm{c}(Y)-$ многозначное отображсение такое, что

(i) $H(\cdot, x) \in G V_{\Phi}(I ; \mathrm{c}(Y))$ для всех $x \in X$;

(ii) $H(t, \cdot): X \rightarrow \mathrm{c}(Y)$ является п.н.св. для всех $t \in I$. 
Тогда существует селекиия $h: I \times X \rightarrow Y$ отображсения $H$ такая, что

(a) $h(\cdot, x) \in G V_{\Phi}(I ; Y)$ для всех $x \in X$;

(b) $p_{\Phi}(h(\cdot, x), I) \leqslant p_{\Phi}(H(\cdot, x), I)$ для всех $x \in X$;

(c) $d\left(\eta(x), h\left(t_{0}, x\right)\right)=\operatorname{dist}\left(\eta(x), H\left(t_{0}, x\right)\right)$ для всеx $x \in X$;

(d) $h(t, \cdot): X \rightarrow Y$ непрерывно для всех $t \in I$.

Если, кроме того, $\sup _{x \in X} p_{\Phi}(H(\cdot, x), I)<\infty$, то селекиия $h$ будет непрерывной на $I \times X$. Отметим, что из (c) вытекает, в частности, что если $\eta$ есть селекиия $H\left(t_{0}, \cdot\right), \operatorname{mo} h\left(t_{0}, \cdot\right)=\eta$.

ДокАЗАТЕЛЬСТво. Обозначим через $C(I ; Y)$ пространство всех непрерьвных отображений из $I$ в $Y$ с компактно открытой топологией, предбазой которой являются множества $\{f \in C(I ; Y) \mid f(J) \subset \mathscr{U}\}$, где $J \subset I$ компактно и $\mathscr{U} \subset Y$ открыто. Определим многозначное отображение $Г$ из $X$ в $C(I ; Y)$ правилом

$$
\begin{gathered}
\Gamma(x)=\left\{f \in C(I ; Y) \mid f(t) \in H(t, x) \quad \forall t \in I, \quad p_{\Phi}(f, I) \leqslant p_{\Phi}(H(\cdot, x), I),\right. \\
\left.d\left(\eta(x), f\left(t_{0}\right)\right)=\operatorname{dist}\left(\eta(x), H\left(t_{0}, x\right)\right)\right\}, \quad x \in X .
\end{gathered}
$$

Для $x \in X$ имеем $\Gamma(x) \neq \varnothing$ в силу теоремы 10 и предположения (i), и $\Gamma(x)$ есть замкнутое подмножество в $G V_{\Phi}(I ; Y)$ благодаря лемме $4($ е). Покажем, что отображение $\Gamma$ действует из $X$ в $c(C(I ; Y))$ и является п.н.св. Действительно, при любом $x \in X$ семейство $\Gamma(x)$ равностепенно непрерывно в силу леммы $4($ a) и условия $\Phi \in \mathscr{N}$, причем для любого $t \in I$ множество $\{f(t) \mid f \in \Gamma(x)\}$ компактно в $Y$ в силу компактности множества $H(t, x)$, поэтому по теореме Арцела-Асколи $\Gamma(x)$ предкомпактно в $C(I ; Y)$, а поскольку множество $\Gamma(x)$ замкнуто, то оно компактно в $C(I ; Y)$. Для доказательства п.н.св. Г в силу замечаний перед леммой 14 достаточно показать, что Г слабо п.н.св. Для $\varepsilon>0, g \in C(I ; Y)$ и $t \in I$ положим

$\overline{\mathscr{U}}_{\varepsilon}(g)=\left\{f \in C(I ; Y) \mid \sup _{t \in I} d(f(t), g(t)) \leqslant \varepsilon\right\} \quad$ и $\quad \bar{B}_{\varepsilon}(g(t))=\{y \in Y \mid d(g(t), y) \leqslant \varepsilon\}$.

Для $x \in X$ обозначим через $\Gamma_{1}(x)$ (компактное) множество отображений $f \in C(I ; Y)$, удовлетворяющих первым двум условиям из определения $\Gamma(x)$, и через $\Gamma_{2}(x)$ - множество $f$, удовлетворяющих третьему условию из определения $\Gamma(x)$. Заметим, что

$$
\Gamma_{1}^{-}\left(\overline{\mathscr{U}}_{\varepsilon}(g)\right)=\left\{x \in X \mid \Gamma_{1}(x) \cap \overline{\mathscr{U}}_{\varepsilon}(g) \neq \varnothing\right\}=\bigcap_{t \in I} H(t, \cdot)^{-}\left(\bar{B}_{\varepsilon}(g(t))\right),
$$

причем любой предобраз под знаком пересечения в силу условия (ii) является замкнутым в $X$. Следовательно, $\Gamma_{1}^{-}\left(\overline{\mathscr{U}}_{\varepsilon}(g)\right)$ замкнуто в $X$, так что многозначное отображение $\Gamma_{1}: X \rightarrow C(I ; Y)$ п.н.св. Поскольку все значения $\Gamma_{1}(x)$ компактны в $C(I ; Y)$ и график многозначного отображения $\Gamma_{2}$ замкнут в $X \times C(I ; Y)$, по теореме 3.1 .8 из [35] заключаем, что отображение $x \mapsto \Gamma(x)=\Gamma_{1}(x) \cap \Gamma_{2}(x)$ является п.н.св.

По лемме 14 Г имеет непрерывную селекцию $\gamma: X \rightarrow C(I ; Y)$. Положим $h(t, x)=$ $\gamma(x)(t), t \in I, x \in X$. Из определения $\Gamma(x)$ и включения $\gamma(x) \in \Gamma(x), x \in X$, вытекают соотношения (a)-(c). Установим (d). Если $t \in I$ и $\mathscr{U} \subset Y$ открыто, то, положив $\mathscr{U}(t)=\{f \in C(I ; Y) \mid f(t) \in \mathscr{U}\}$, найдем, что

$$
\{x \in X \mid h(t, x) \in \mathscr{U}\}=\{x \in X \mid \gamma(x)(t) \in \mathscr{U}\}=\{x \in X \mid \gamma(x) \in \mathscr{U}(t)\} .
$$


Поскольку $\mathscr{U}(t)$ открыто в $C(I ; Y)$ и $\gamma$ непрерьвно, последнее множество открыто в $X$, поэтому отображение $h(t, \cdot)$ непрерывно для любого $t \in I$.

Если дополнительно известно, что $\sup _{x \in X} p_{\Phi}(H(\cdot, x), I)<\infty$, то построенная вьше селекция $h: I \times X \rightarrow Y$ непрерьвна. Это следует из пункта (b), леммы 4(a), условия $\Phi \in \mathscr{N}$ и следующего неравенства для $\left(t_{0}, x_{0}\right),(t, x) \in I \times X:$

$$
\begin{aligned}
d\left(h(t, x), h\left(t_{0}, x_{0}\right)\right) & \leqslant d\left(h(t, x), h\left(t_{0}, x\right)\right)+d\left(h\left(t_{0}, x\right), h\left(t_{0}, x_{0}\right)\right) \\
& \leqslant \omega_{\Phi}\left(\left|t-t_{0}\right|\right) p_{\Phi}(H(\cdot, x), I)+d\left(h\left(t_{0}, x\right), h\left(t_{0}, x_{0}\right)\right) .
\end{aligned}
$$

Отметим, что существование селекций $h$ со свойством $h(\cdot, x) \in \operatorname{Lip}(I ; Y)$ в случае, когда $H(\cdot, x) \in \operatorname{Lip}(I ; \mathrm{c}(Y))$ в пункте (i) теоремы 15, установлено в работе [16, теорема 5].

Автор выражает признательность рецензенту за полезные советы, способствовавшие некоторому обобщению теоремы 10 , и указание на ссылку [31].

\section{СПИСОК ЦИТИРОВАННОЙ ЛИТЕРАТУРЫ}

[1] Michael E. A. Continuous selections. I // Ann. Math. 1956. V. 63. № 2. P. 361-382.

[2] Kuratowski K., Ryll-Nardzewski C. A general theorem on selectors // Bull. Acad. Polon. Sci. Sér. Sci. Math. Astronom. Phys. 1965. V. 13. P. 397-403.

[3] Чобан М. М. Многозначные отображения и борелевские множества. I // Тр. ММО. 1970. T. 22. C. $229-250$.

[4] Castaing C., Valadier M. Convex Analysis and Measurable Multifunctions. Lecture Notes in Math. V. 580. Berlin: Springer-Verlag, 1977.

[5] Реповш Д., Семенов П. В. Теория Э. Майкла непрерьвных селекций. Развитие и приложения // УМН. 1994. Т. 54. № 6. С. 49-80.

[6] Hermes H. Existence and properties of solutions of $\dot{x} \in R(t, x)$ // Stud. in Appl. Math., SIAM Publications. 1969. V. 5. P. 188-193.

[7] Hermes H. On continuous and measurable selections and the existence of solutions of generalized differential equations // Proc. Amer. Math. Soc. 1971. V. 29. № 3. P. 535-542.

[8] Kikuchi N., Tomita Y. On the absolute continuity of multifunctions and orientor fields // Funkcial. Ekvac. 1971. V. 14. №3. P. 161-170.

[9] Zhu Qiji. Single-valued representation of absolutely continuous set-valued mappings // Kexue Tongbao. 1986. V. 31. №7. P. 443-446.

[10] Мордухович Б. Ш. Методы аппроксимаций в задачах оптимизации и управления. М.: Наука, 1988.

[11] Chistyakov V.V. On mappings of bounded variation // J. Dynam. Control Systems. 1997. V. 3. №2. P. 261-289.

[12] Чистяков В. В. К теории многозначных отображений ограниченной вариации одной вещественной переменной // Матем. сб. 1998. Т. 189. № 5. С. 153-176.

[13] Чистяков В.В. Об отображениях ограниченной вариации со значениями в метрическом пространстве // УМН. 1999. Т. 54. № 3. С. 189-190.

[14] Чистяков В.В.Отображения ограниченной вариации со значениями в метрическом пространстве: обобщения // Труды международной конфереренции, посвященной 90-летию со дня рождения Л. С. Понтрягина. Ч. 2. Негладкий анализ и оптимизация. Итоги науки и техники. Современная математика и ее прилож. Т. 61. М.: ВИНИТИ, 1999. С. 167-189.

[15] Chistyakov V. V. Generalized variation of mappings with applications to composition operators and multifunctions // Positivity. 2001. V. 5. № 4. P. 323-358.

[16] Ślęzak W. A. Concerning continuous selectors for multifunctions with nonconvex values // Zeszyty Nauk. WSP Bydgoszcz. Problemy Matematyczne. 1987. V. 9. P. 85-104.

[17] Belov S. A., Chistyakov V. V. A selection principle for mappings of bounded variation // J. Math. Anal. Appl. 2000. V. 249. №2. P. 351-366. 
[18] Chistyakov V. V. Generalized variation of mappings and applications // Real Anal. Exchange. 1999-2000. V. 25. № 1. P. 61-64.

[19] Maligranda L. Orlicz Spaces and Interpolation. Seminars in Math. V. 5. Brasil: Univ. of Campinas, IMECC-UNICAMP, 1989.

[20] Красносельский М. А., Рутицкий Я. Б. Выпуклые функции и пространства Орлича. М.: Физматгиз, 1958.

[21] Натансон И. П. Теория функций вещественной переменной. М.: Наука, 1974.

[22] Шварц Л. Анализ. Т. 1. М.: Мир, 1972.

[23] Рисс Ф., Сёкефальви-Надь Б. Лекции по функциональному анализу. М.: Мир, 1979.

[24] Медведев Ю. Т. Обобщение одной теоремы Ф. Рисса // УМН. 1953. Т. 8. №6. С. 115-118.

[25] Cybertowicz Z., Matuszewska W. Functions of bounded generalized variations // Comment. Math. Prace Mat. 1977. V. 20. P. 29-52.

[26] Maligranda L., Orlicz W. On some properties of functions of generalized variation // Monatsh. Math. 1987. V. 104. P. 53-65.

[27] Komura Y. Differentiability of nonlinear semi-groups // J. Math. Soc. Japan. 1969. V. 21. P. 375-402.

[28] Helly E. Über lineare Funktionaloperationen // Sitzungsberichte der Naturwiss. Klasse. Kais. Akad. Wiss. (Wien). 1912. V. 121. P. 265-297.

[29] Kupka I. Continuous multifunction from $[-1,0]$ to $\mathbb{R}$ having no continuous selection // Publ. Math. Debrecen. 1996. V. 48. № 3-4. P. 367-370.

[30] Chistyakov V. V., Galkin O. E. On maps of bounded $p$-variation with $p>1 / /$ Positivity. 1998. V. 2. №1. P. 19-45.

[31] Guričan J., Kostyrko P. On Lipschitz selections of Lipschitz multifunctions // Acta Math. Univ. Comenian. 1985. V. 46/47. P. 131-135.

[32] Куратовский К. Топология. Т. 2. М.: Мир, 1969.

[33] Hasumi M. A continuous selection theorem for compact-valued maps // Math. Ann. 1969. V. 179. P. 83-89.

[34] Graf S. A measurable selection theorem for compact-valued maps // Manuscripta Math. 1979. V. 27. P. 341-352.

[35] Обен ЖК.-П., Экланд И. Прикладной нелинейный анализ. М.: Мир, 1988.

Нижегородский государственный университет им. Н. И. Лобачевского

Поступило

E-mail : chistya@mm.unn.ac.ru

02.02 .2000

Исправленный вариант

09.02 .2001 